\title{
UBVRI simultaneous observations of the nucleus of Seyfert galaxy NGC 5548 in 1993-1999
}

\begin{abstract}
N. I. Merkulova ${ }^{\star}$
Crimean Astrophysical Observatory, Crimea, Ukraine; and Isaac Newton Institute of Chile, Crimean Branch, 98409, p/o Nauchny, Crimea, Ukraine

Received 11 May 2000 / Accepted 20 February 2002

Abstract. An ongoing program on photometric and spectral monitoring of some bright Seyfert galaxies has been carried out at the Crimean Astrophysical Observatory since 1989. Results of photometric observations of NGC 5548 obtained with the $1.25 \mathrm{~m}$ telescope are reported in this paper; it focuses on the analysis of intranight variations. During 44 observational nights in 1993-1999 in each spectral band of the Johnson UBVRI system, 672 measurements have been performed simultaneously through the round aperture (diameter $15^{\prime \prime}$ ) using differential photometry techniques. The estimated accuracy of each measurement is about 0.01 mag. The peak amplitude $R_{\max }=F_{\max } / F_{\min }=2.99$ for the whole light curve was observed in the $U$ band, while the minimum amplitude $F_{\max } / F_{\min }=1.37$ occurred in the $I$ band during the full observation period. $U B V R I$ observations and good sampled data of international monitoring campaigns of NGC 5548, were used to calculate Structure Functions. A comparison is made of the characteristics of the long and short time scale variations of NGC 5548 with those of NGC 4151, NGC 7469 and NGC 1275. In order to examine the intranight variations of the nucleus of NGC 5548, standard deviations $(S D)$ of the nightly averaged flux $F$, and a measure of intranight variability $-S D / F$ were calculated for each night. Using this parameter, a probability characteristics is introduced, and duty cycles (the fraction of time when the galaxy is variable), characterizing the efficiency of the central energy source, were evaluated. It is concluded that intranight variability is really transient in character and manifests itself with different probabilities for different galaxies.
\end{abstract}

Key words. galaxies: Seyfert: photometry - galaxies: individual: NGC 5548

\section{Introduction}

Seyfert Galaxy nuclei (Sy) together with quasi-stellar objects (QSO), BL Lac type objects (Lacertides and Blazars) and some others are part of the broad class of objects called Active Galactic Nuclei (AGN). It is probable, that all these objects are different versions of the same phenomenon. Variability on different timescales throughout the entire electromagnetic spectrum is a common property among AGNs. The circumnuclear region cannot be resolved spatially, so a productive way to investigate nuclear phenomena is to study continuum and line variability, especially on short timescales. One can obtain important information on the structure and kinematics of this region by examining how the broad-line profiles change in response to changes in the continuum flux (this is the so-called "reverberation mapping" technique, see Blandford \& McKee 1982 and Capriotti et al. 1982). A cross-correlation function (CCF) between the continuum and emission-line light curves is a measure of the variable part of broad-line region (BLR), see Koratkar \& Gaskell (1991). To detect accurately a time delay between a change in the ionizing continuum and a corresponding change in the emission-line flux is one of the goals of the International AGN Watch project; closely spaced multiwavelength observations of several bright Seyfert 1 galaxies have been obtained during the past decade (see, for instance, Rodriguez-Pascual et al. 1997).

One of the main targets for AGN Watch campaigns is the low-redshift $(z=0.0168)$ Seyfert 1 galaxy NGC 5548 (Clavel et al. 1991). The optical continuum variability of this nucleus was first reported by Deych (1966): an increase of the galaxy brightness of $0.5 \mathrm{mag}$ in the period from 1940 to 1956 was registered using photographic plates. Lyuty (1972) detected $U$ band brightness variations up to $0.7 \mathrm{mag}$ in 250 days.

The results of the ground-based observations obtained during a period of 8 years in the frame of the AGN Watch project showed that the nuclear continuum in the optical has varied by a factor of more than 7 , and the $\mathrm{H}_{\beta}$ emission line has varied by a factor of nearly 6 (Peterson et al. 1999). The time lag between variations of the continuum flux and the $\mathrm{H}_{\beta}$ line is $\sim 10-20$ days, varying somewhat in the sense that the lag is larger when the source

\footnotetext{
* e-mail: nelly@crao.crimea.ua
} 
is brighter. During the multiwavelength monitoring campaign, the $1350 \AA$ continuum flux was found to have varied by a factor of 2 ; the variability amplitude was larger at shorter wavelengths, and more short time scale variability was observed (probably) at shorter wavelengths (Korista et al. 1995). Dumont et al. (1998) modeled the BLR in NGC 5548 as a 3 -zone region where the location of the various components was determined by the mean values of the line-to-continuum lag.

The existence of small variations in brightness at different frequencies ("microvariability") is now a well established fact for the most luminous active nuclei of a BL Lac type and for highly polarized quasars. The most rapid rate of change detected $(0.06 \pm 0.01 \mathrm{mag} / \mathrm{hour})$ was reported by Carini et al. (1991) for a BL Lac object, AP Librae. The clearest detection of microvariability (but not the only one) obtained during a two year intensive monitoring of a BL Lac object 3C $66 \mathrm{~A}$ was a 0.2 mag decline in the brightness in 6 hours (Takalo et al. 1996). The authors did not see microvariability during each night's monitoring, thus they claimed that the microvariability in $3 \mathrm{C} 66 \mathrm{~A}$ (and perhaps in all blazars) is a randomly occurring event. They suspected that the mechanisms for different time scale variations are not connected: the flares and outbursts are caused by shocks in the jet and the microvariability (perhaps) by instabilities in the accretion disk.

In the case of Seyfert galaxies the existence of microvariability is still under debate. This is probably connected with the fact that Seyfert galaxies do not show variability on a detectable level every night. Intranight variations of the type 1 Seyfert galaxy NGC 4151 are reported by Lyuty et al. (1989) only on 8 observation nights out of 13 . The accuracy of observations performed in the $V$ band was $\leq 1 \%$. Variations with an amplitude of $\sim 0$. 010 and time scale of $15-30$ min were detected on some nights. They also concluded that manifestation of rapid variability is (perhaps) independent of mean galaxy brightness.

The shortest time scale of variations in the optical was reported by Dultzin-Hacyan et al. (1992) for the NGC 7469 galaxy nucleus. Using a Stromgren photometer, they investigated variations with a duration of $\sim 13 \mathrm{~min}$ and average amplitude $\sim 0$. 040 (at a $\sigma \geq 4.5$ level) within a $20^{\prime \prime}$ diaphragm during the first four out of 5 photometric nights $(\sim 29 \mathrm{hr})$ of the observing run on August 1990. During the fifth night the variability nearly disappeared. A very thorough analysis of errors was reported.

New results on the multiwavelength variability of AGN obtained over the past decade can be found in the review by Ulrich et al. (1997) together with a discussion of all available models for energy sources producing variable fluxes. They pointed out that the intranight continuum variability in the optical is generally small, with only a weak tendency for larger amplitude variability at shorter wavelength. The best current model for the rapidly variable continuum emission is reprocessed emission from a dense medium irradiated by variable X-rays.

An ongoing program on photometric and spectral monitoring of some bright Seyfert galaxies has been carried out at the Crimean Astrophysical Observatory since 1989. The results of photometric observations of NGC 5548 are reported in this paper; it focuses on the analysis of the intranight variations. A comparison is made of the characteristics of long and short time scale variations of NGC 5548 with those of NGC 4151, NGC 7469 and NGC 1275.

\section{Observations and reductions}

Accurate multicolor $U B V R I$ photometry has been carried out with the $1.25 \mathrm{~m}$ telescope at the Crimean Astrophysical Observatory using a five--channel version of the Double Image Chopping Photometer-Polarimeter by Prof. V. Piirola (1973).

The observations were made simultaneously in five colors using dichroic mirrors to split the light into five spectral bands. The resulting passbands are close to the standard Johnson's $U B V R I$ photometric system, with effective wavelengths of $0.36,0.44,0.53,0.69$ and $0.83 \mu \mathrm{m}$, respectively, corresponding to values of $\log \nu(U)=14.914$, $\log \nu(B)=14.832, \log \nu(V)=14.756, \log \nu(R)=14.643$, $\log \nu(I)=14.558$ in frequency units $(\mathrm{Hz})$ used throughout the paper.

In the focal plane of the 1.25-meter telescope there is a diaphragm with two equal apertures (circular apertures $20^{\prime \prime}, 15^{\prime \prime}$ or $10^{\prime \prime}$ in diameter are used). The distance between the centers of the apertures is $26^{\prime \prime}$. The light of the observed galaxy (star) passes through one of the apertures while the other samples the background. A rotating chopper alternately closes each of the apertures. Thus the photocathode is illuminated alternately by the galaxy (or the star) and the sky background. Because the centers of the apertures are closely spaced, special observations of the pure background at a distance of about $7^{\prime}$ from the galaxy nucleus are added in order to correct the background observations for a contribution from the outer regions of the galaxy.

The telescope is fully automated: during observations an autoguider is employed. As a rule, the galactic nucleus was observed on nights with good atmospheric conditions (estimated seeing between $1^{\prime \prime}-5^{\prime \prime}$ ). The object positioning during observation is done by the autoguider with an accuracy of better than one-fifth of the estimated seeing, i.e. better than $1^{\prime \prime}$ in the worst case. The positioning of the galaxy nucleus in the aperture is done by the autoguider, too, using the guide star, and to within the same positioning errors.

In order to estimate the influence of errors from possible miscentering and/or misguiding of the nucleus, a special test observation was made of the NGC 7469 nucleus (see Fig. 4 in the paper by Merkulova 2000). The result of this guiding test is very near to the calculations that have been made by Dultzin-Hacyan et al. (1992): the measured brightness has an appreciable error (more than 1\%) at a distance more than $5^{\prime \prime}$ from the center of a $20^{\prime \prime}$ diaphragm.

A conventional technique of differential measurements of variable stars was used. Two comparison stars (labeled 


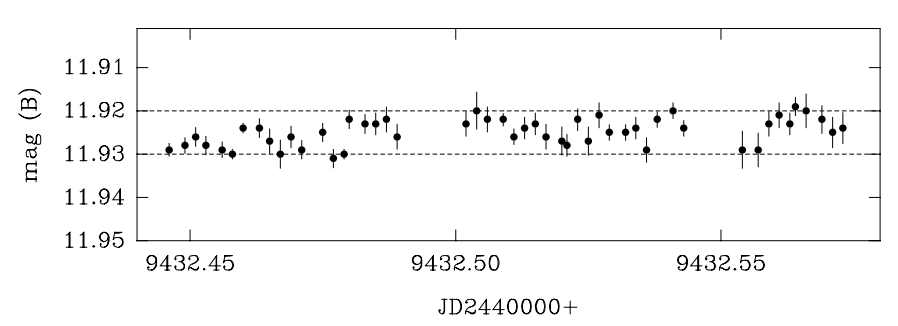

Fig. 1. The light curve of NGC 4151 obtained with a circular diaphragm $15^{\prime \prime}$ in diameter in the $B$-band over the night JD 2449432. The galaxy brightness was constant to within about $1 \%$ over this night. Bars are rms variations about the averages obtained from 8 integrations.

as C1 and C) were taken from the list of Lyuty (1972). Observations were performed against comparison star $\mathrm{C} 1$; $\mathrm{C}$ was used for check purposes. The re-estimated magnitudes of a comparison star $\mathrm{C} 1$ are as follows: $U=$ 13 . $228 \pm 0.018, B=12^{\mathrm{m}} \cdot 780 \pm 0.012, V=11 . \mathrm{m} 904 \pm 0.010$, $R=11^{\mathrm{m}} 173 \pm 0.008$, and $I=10{ }^{\mathrm{m}} 642 \pm 0.008$. Secondary $U B V R I$ standards by Neckel \& Chini (1980) were used for this purpose.

As a rule, observations are carried out in the following sequence: $\mathrm{C}-\mathrm{C}_{1}-$ sky $-\mathrm{AGN}-$ sky $-\mathrm{C}_{1}-\mathrm{C}-$ sky, etc. The time resolution was about 3.5 min. During each observation 8 integrations of $10 \mathrm{~s}$ each were made. The data reduction was done using the standard photometry reduction package. Photon statistics (corrected for sky background) were applied to calculate photometric errors, which should be the same as rms errors obtained by averaging 8 integrations. Taking into account the variations of the background and comparison star, we derived rms errors of a single observation in each of the $U B V R I$ bands, of $0{ }^{\mathrm{m}} 016,0{ }^{\mathrm{m}} 017,0{ }^{\mathrm{m}} 022,0{ }^{\mathrm{m}} 012$, and $0^{\mathrm{m}} 020$, respectively. Therefore, the rms errors of a mean observation consisting of a series of 8 measurements are equal to $\sigma=0$. 006 , 0. $006,0.008,0.004$, and 0.007 , respectively.

Similar accuracies of about 1 percent are claimed for other Seyfert galaxy nuclei observed in the frame of the photometric monitoring program. This reported level of accuracy needs some comments:

1) As is known, Seyfert galaxies do not show their variability every night. So, the author searched for a long night when a Seyfert galaxy showed a light curve that was constant to within about 1 percent. The observations of the NGC 4151 nucleus are best sampled. During the night of JD 2449432 the fluxes in all bands were almost constant, especially in the $B$-band (see Fig. 1). From this figure, the brightness in the $B$-band can be seen to be constant to within about 1 percent.

2) In order to test the observational technique used for the observations of all Seyfert galaxies reported here, a special observation was made using comparison stars from the list by Luytyi (1972) for the galaxy NGC 1275: instead of the galaxy nucleus the author used the star C2, and observations over the night 24/25 December, 2000 (JD 2451903) were made using C1 as a standard star. The background was measured at the same distance as it was

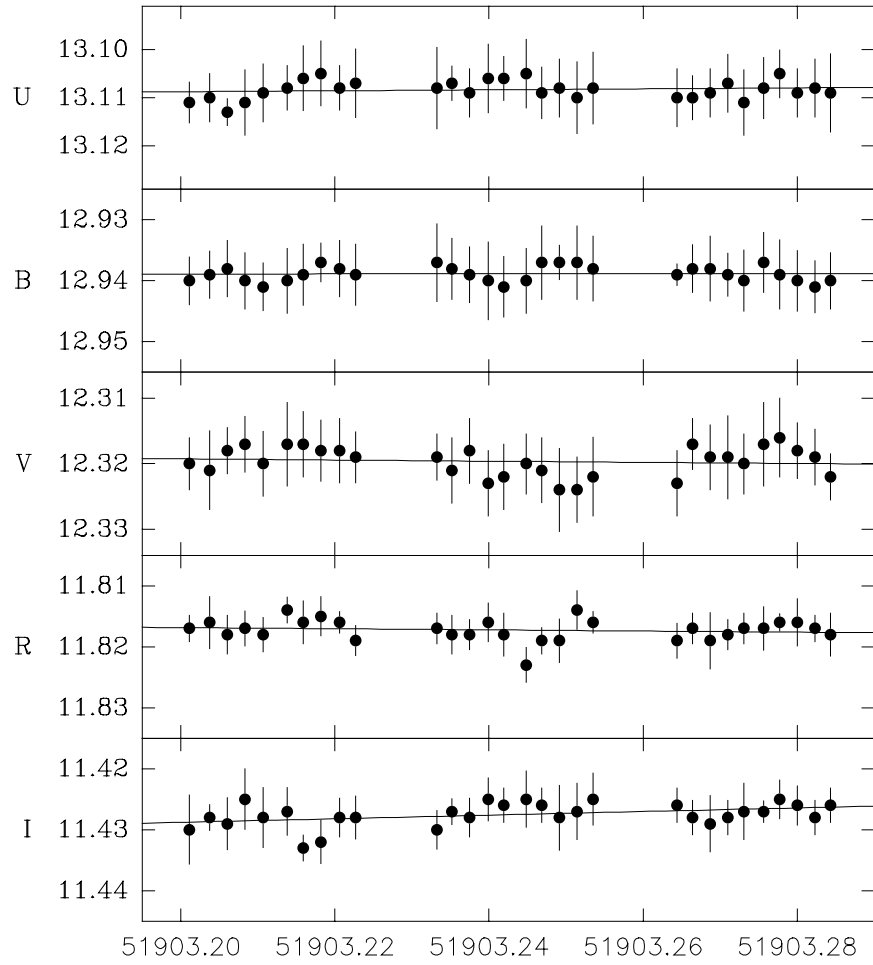

Fig. 2. The light curve of the comparison star $\mathrm{C} 2$ for the galaxy NGC 1275 which was observed as "a galaxy" in a standard manner using a circular diaphragm $15^{\prime \prime}$ in diameter. The observations were processed using a differential technique, against the standard star $\mathrm{C} 1$. The boxes are mean magnitudes obtained by averaging 8 integrations plotted against Julian date of midobservation for the $U B V R I$ bands, respectively, from top to bottom. Bars are rms variations about these averages.

during the real observation of this galaxy nucleus. The results of this observation can be seen in Fig. 2 .

From this figure, all data are within the error bars, and nightly mean values for the star $\mathrm{C} 2$ are as follow: $U=$ $13 . \mathrm{m} 108 \pm 0.002, B=12^{\mathrm{m}} 939 \pm 0.001, V=12^{\mathrm{m}} 320 \pm 0.002$, $R=11.817 \pm 0.002$, and $I=11.428 \pm 0.002$.

In total, 672 observations of the NGC 5548 nuclear region were obtained over 44 nights on 7 observing runs from 1993, March 22 to 1999, July 10 (JD 2449069JD 2451370), using a circular aperture $15^{\prime \prime}$ in diameter. The intranight observations lasted from 0.5 up to $3^{\mathrm{h}} .5$. The net on-source integration time was about 47 hours, in total. All dates with number of observational points $n \leq 4$ were excluded from the sample analyzed.

\section{UBVRI light curves of NGC 5548 in 1993-1999}

Stellar magnitudes were transformed into fluxes in $\mathrm{mJy}$ $\left(1 \mathrm{mJy}=10^{-29} \mathrm{~W} / \mathrm{m}^{2} \mathrm{~Hz}\right.$ ), using standard procedures (O'Dell et al. 1978). The observations were averaged by night; the light curves in the $U B V R I$ bands are shown in Fig. 3. Vertical bars are rms errors of nightly averaged values. 


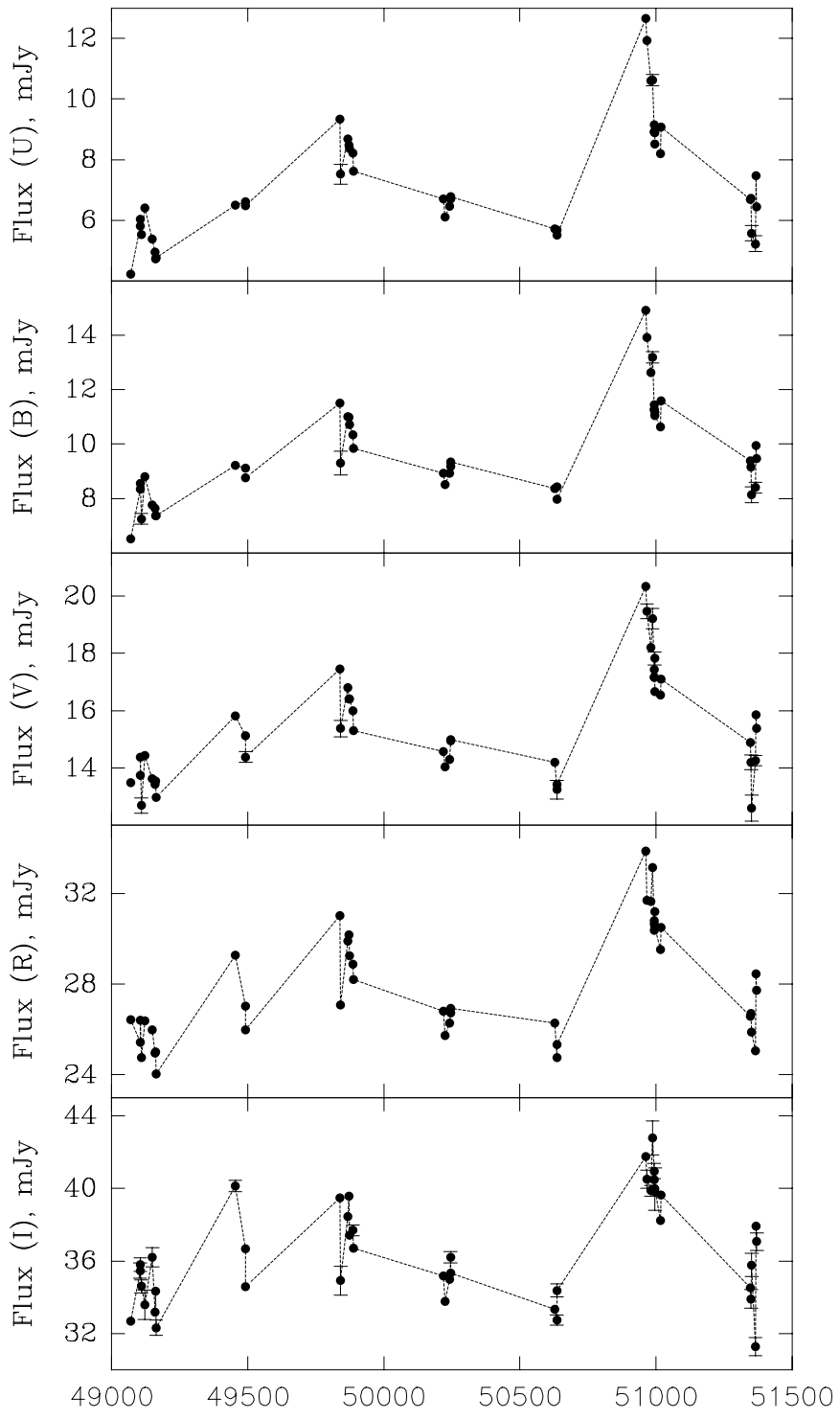

Fig. 3. The light curves of NGC 5548 obtained with the circular diaphragm $15^{\prime \prime}$ in diameter. The boxes are nightly mean values of flux densities in mJy against Julian date of midobservation for the $U B V R I$ bands respectively, from top to bottom. Bars are rms variations about these averages.

Unfortunately, these observations are not well sampled. Two parts of the data reported here were used in two papers written when analyzing two monitoring campaigns of the NGC 5548 nucleus (Korista et al. 1997; Dietrich et al. 2001). In both cases the data obtained by the author coincided well with the other data. Figure 4 shows the comparison of our observations with a part of the best sampled data obtained in the framework of the International AGN Watch monitoring (Peterson et al. 1999). Unfortunately, these data are not multiwavelength; only fluxes at $\lambda 5100 \AA$ in $\operatorname{ergs~cm}{ }^{-2} \mathrm{~s}^{-1} \AA^{-1}$ are available. These data reduced to their average are shown by dots in Fig. 4. Fluxes obtained by the author in the $V$ band are expressed in $\mathrm{mJy}$; they were reduced to their average, too, and are shown by stars in Fig. 4 .

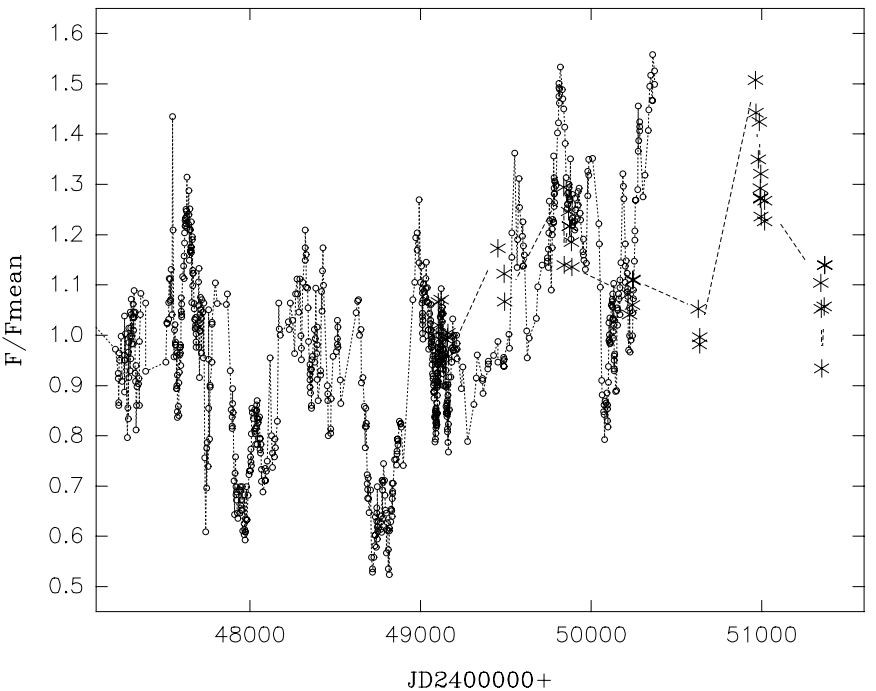

Fig. 4. Comparison of two samples of the NGC 5548 nucleus fluxes: a part of the best sampled data obtained during AGN Watch monitoring are shown by open circles, and fluxes obtained by the author in the $V$ band are shown by stars. All data are reduced to their averages.

Observations of the nucleus of NGC 5548 were compared with analogous data samples of other Seyfert galaxies NGC 4151, NGC 7469 and NGC 1275. The homogeneity of data obtained with the same telescope using the same technique has made statistical comparisons possible among different galaxies and different wave bands. The following samples were used for the analysis:

1) Observations of the NGC 4151 nucleus obtained over 96 nights in 1989-1996. More than 1500 measurements were done simultaneously in each spectral band using the same technique as described in this paper (Merkulova et al. 1999). The light curve of NGC 4151 in each of $U B V R I$ bands shows a slow increase of intensity during almost the entire observation period, overlapped by rapid outbursts.

2) An analogous sample for NGC 1275 (a BL Lac type object) consisting of 820 measurements performed simultaneously in 5 bands over 37 nights. The data have been published by Pronik et al. (1999a). From the late 70ies the nucleus has been at minimum brightness.

3) Observations of the nuclear region of the Seyfert galaxy NGC 7469 obtained over 64 nights in 1990-1998 consisting of about 1500 measurements (Merkulova 2000). During the observing period the mean luminosity of the nucleus was almost constant; only overlapping brightness fluctuations were observed.

For each sample, the estimated accuracy of a single measurement was about $0 \mathrm{~m}^{\mathrm{m}} 01$, the monitoring over one night lasted from 0.5 to $\sim 5.5$ hours, the time resolution was about 3.5 min. Data on the galaxies and the data samples in question are combined in Table 1; summarized as follows:

Column (1) - name and type of the object, (2) - start and end date of the observing period, 
Table 1. Seyfert galaxies and their data samples discussed in the paper.

\begin{tabular}{|c|c|c|c|c|c|c|c|c|}
\hline $\begin{array}{l}\text { Object, } \\
\text { type }\end{array}$ & $\begin{array}{l}\text { Observing } \\
\text { period }\end{array}$ & $\begin{array}{l}\text { Duration } \\
\text { in years }\end{array}$ & $\begin{array}{l}\text { No. of } \\
\text { nights }\end{array}$ & $\begin{array}{l}\text { No. of } \\
\text { runs }\end{array}$ & $\begin{array}{c}\text { Redshift } \\
\text { z }\end{array}$ & $\begin{array}{c}\text { Distance } \\
\left(H_{0}=75 \mathrm{~km} \mathrm{~s}^{-1} / \mathrm{Mpc}\right)\end{array}$ & $\begin{array}{l}\text { Aperture, } \\
\text { arcsec }\end{array}$ & $\begin{array}{l}\text { Aperture } \\
\text { dimension }\end{array}$ \\
\hline 1 & 2 & 3 & 4 & 5 & 6 & 7 & 8 & 9 \\
\hline $\begin{array}{l}\text { NGC } 1275 \\
\text { BL Lac }\end{array}$ & $\begin{array}{l}22 \text { Dec. } 1989- \\
29 \text { Dec. } 1994\end{array}$ & $4.7 \mathrm{y}$ & 37 & 5 & 0.0178 & $71 \mathrm{Mpc}$ & 20 & $6.9 \mathrm{kpc}$ \\
\hline $\begin{array}{l}\text { NGC } 4151 \\
\quad \text { Sy1 }\end{array}$ & $\begin{array}{l}11 \text { Feb. } 1989- \\
14 \text { Jun. } 1996\end{array}$ & $7.5 \mathrm{y}$ & 96 & 8 & 0.0033 & $14 \mathrm{Mpc}$ & 20 & $1.4 \mathrm{kpc}$ \\
\hline $\begin{array}{l}\text { NGC } 5548 \\
\quad \text { Sy1 }\end{array}$ & $\begin{array}{l}22 \text { Mar. } 1993- \\
10 \text { Jul. } 1999\end{array}$ & $5.8 \mathrm{y}$ & 44 & 7 & 0.0168 & $67 \mathrm{Mpc}$ & 15 & $4.9 \mathrm{kpc}$ \\
\hline $\begin{array}{l}\text { NGC } 7469 \\
\quad \text { Sy1 }\end{array}$ & $\begin{array}{l}24 \text { Sep. } 1990- \\
22 \text { Oct. } 1998\end{array}$ & $7.9 \mathrm{y}$ & 64 & 9 & 0.017 & $68 \mathrm{Mpc}$ & 20 & $6.6 \mathrm{kpc}$ \\
\hline
\end{tabular}

(3) - duration of the observing period in years, (4) - number of observation nights, (5) - number of observing runs, (6) - redshift $z,(7)$ - galaxy distance (the Hubble constant $H_{0}=75 \mathrm{~km}^{-1} \mathrm{~s}^{-1} \mathrm{Mpc}^{-1}$ throughout the paper), (8) - aperture used in the observations, (9) - aperture projection onto sky.

A parameter $R_{\max }$ (ratio of the maximum flux $F_{\max }$ to the minimum one $F_{\text {min }}$ ) was calculated for the whole observation period of the NGC 5548 nucleus. In Fig. 5, the ratios $R_{\max }=F_{\max } / F_{\min }$ are shown for the different wave bands (filled circles). One can see that the peak amplitude $F_{\max } / F_{\min }=2.99$ on the light curve was observed in the $U$ band, while the minimum amplitude $F_{\max } / F_{\min }=1.37$ was in the $I$ band during the whole observation period.

The variability parameter $R_{\max }$ of the Sy1 galaxy NGC 7469 is shown in Fig. 5 by open circles. This parameter was obtained from the $U B V R I$ observations for the period 1990-1998 only (Merkulova 2000). The photometric behavior of these two galaxies was almost identical. But the variability amplitudes in the $U$ and $B$ bands are higher for NGC 5548, and they are almost equal for $V, R, I$ bands. It is clear that the amplitude of long-term variations is higher in the UV region than in the near IR, where stellar contamination is higher.

A new cycle of nuclear activity observed in NGC 4151 (filled triangles) distinguished this Sy1 galaxy from the other two Sy1, especially in the UV region. It is interesting that NGC 1275 shows long-term variations on a time scale of years (open triangles), which are almost identical in all wave bands.

\section{Structure function of the variable optical fluxes of the nucleus of NGC 5548}

A formal technique to analyze light curves involves the computation of the Structure Function $(S F)$ discussed by Simonetti et al. (1985). The first-order $S F$ is defined as:

$S F(\mathrm{~d} t)=<[F(t)-F(t+\mathrm{d} t)]^{2}>$,

where $F(t)$ and $F(t+\mathrm{d} t)$ are observed fluxes separated by time interval $\mathrm{d} t$, and the angular brackets denote an ensemble average. The $S F$ of an "ideal" stationary random

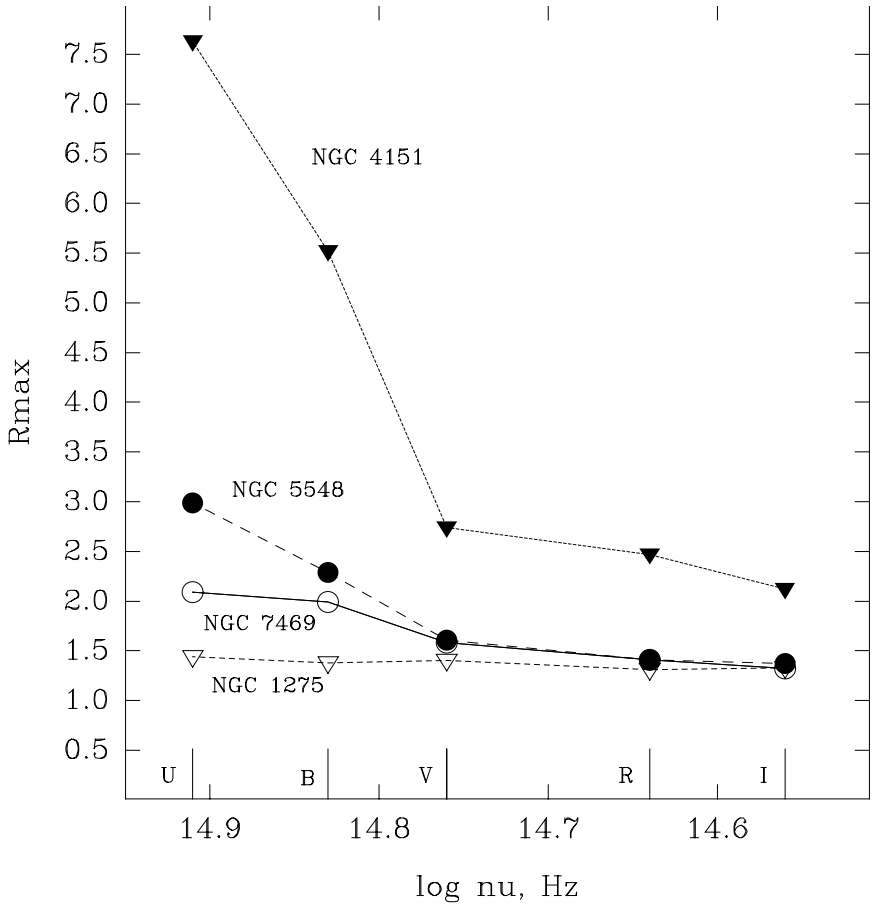

Fig. 5. Variability amplitude $R_{\max }=F_{\max } / F_{\min }$ for each of the $U B V R I$ bands for the whole period of the observations of 4 galaxies: NGC 5548 (filled circles), NGC 7469 (open circles), NGC 4151 (filled triangles), and NGC 1275 (open triangles). The locations of $U B V R I$ wave bands are given by vertical lines in the abscissa.

process on a logarithmic scale consists of three components: a slope

$b=\mathrm{d} \log (S F) / \mathrm{d} \log \mathrm{d} t$,

which is located between two plateaus. The logarithmic slope " $b$ " characterizes the nature of the process: $b=0$ corresponds to flicker-noise, $b=1$ - to shot-noise. It is well known (Press 1978), that three types of noise spectra are relevant for variability in AGNs: random or white noise, shot noise or random-walk noise, and flicker noise. There is no "memory" for white noise, i.e. the next point is unrelated to the preceding one. Shot noise has an infinite memory of a preceding event. Flicker noise is halfway between white and shot noise and has a finite memory 
in the sense that the next point depends on the preceding values, although with a decreasing dependence upon events further away from the current time. The longest correlation time scale $-T_{\max }$ characterizes the duration of the flares. Thus, this technique can give an indication that all timescales have been resolved by the curve "turning over" (Hufnagel \& Bregman 1992). An SF analysis gives information similar to a power spectrum analysis, but the $S F$ has advantages when examining nonperiodic data, because there are no problems of windowing and aliasing. It provides more readily comprehensible information on the timescales, and is more successful in determining the noise processes. It excludes the influence of stellar contamination on the observed flux, because of the differences in fluxes used in $S F$ calculations. In the application to AGNs the Structure Function was discussed by Hufnagel \& Bregman (1992), Hughes et al. (1992), Lainela \& Valtaoja (1993), Smith et al. (1993), and others.

A special program package by S. G. Sergeev has been used for the Structure Function calculations. The codes were tested using observations of the quasar 3C 273. The results of the test allow us to conclude that the program package works well (Pronik et al. 1999b). Optical observations of the quasar 3C 273 during 1887-1967 reduced in a common system by Kunkel (1967) and given in arbitrary units by Fahlmann \& Ulrich (1975) were averaged over 100 days intervals and used for testing. The $S F$ was calculated, and the maximum time of correlated variability $T_{\max }$ was evaluated equal 3.2 years; this corresponds to a logarithmic slope $b=0.98$. The light curve of this quasar was investigated with a correlation method and power spectra analysis by Kunkel (1967), Terrel \& Olsen (1970), Terebizh (1993) and others. According to these investigations, the power spectrum of the variable flux of $3 \mathrm{C} 273$ is $g(\nu) \sim \nu^{-2}$. The average duration of one flare equals 3.2 years according to Terrel \& Olsen (1970), and 2.7 years according to Terebizh (1993). There is a simple correspondence between the power spectrum of the Fourier analysis and the $S F$ analysis: if $S F(\mathrm{~d} t) \sim \mathrm{d} t^{b}$, then $g(\nu) \sim \nu^{-(b+1)}$; taking into account this correspondence, one can obtain the power spectrum using the slope "b" of the $S F$ in the form $g(\nu) \sim \nu^{-1.98}$. This shows that the evaluated characteristics obtained from the $S F$ analysis are in good agreement with the data calculated by other authors using different methods.

The Structure Function is calculated by binning time lags; bins are evenly spaced in time on a logarithmic scale. The value of the Structure Function was calculated for $n_{i}$ pairs falling into an $i$ th bin with time lag $\mathrm{d} t\left(n_{i} \geq 4\right)$. The errors on the $S F$ calculations are equal to the errors of the mean value of the $S F$ calculated for the $i$ th bin. Thus, it is possible to evaluate confidence levels of the $S F$ for each bin, using the $\chi^{2}$-test.

For clarification Fig. 6 shows the $S F$ for the variable flux of NGC 5548 in 1993-1999 for the $U$ band (top panel) only. One can see that there is no $S F$ of a simple "ideal" one-process form with one slope for all time lags. The slopes " $b$ " of the $S F$ for the intranight and years variations are essentially different. Moreover, intranight variability in different bands is characterized by different slopes and different values of $T_{\max }$, i.e. there are flares present with different durations. Parameters of $S F$ s for long-term and intranight variations should be considered separately. Therefore, a regression line on the $S F$ is indicated only for long-term variations.

The observations of the nucleus of NGC 5548 by the author are poorly sampled; it would be interesting to use better sampled data, for example, data of the International AGN Watch campaigns. These data are badly sampled on time scales less than 1 day because only 2-3 measurements were obtained per night, and data obtained by different authors were combined and intercalibrated. But on time scales of more than 1 day an unprecedented sampling has been obtained: a typical spacing is a few days between observations (Peterson et al. 1999). A sample of "pre-AGN Watch" intercalibrated data from the paper by Sergeev et al. (1997) was used as well. The whole sample analyzed here contained 1096 measurements obtained over 19 years in the period from 1977 (JD 2443216) to 1996 (JD 2450373) by different authors; the data were intercalibrated thoroughly, and were taken from papers by Peterson et al. (1999) and by Sergeev et al. (1997). Unfortunately, these data are not multiwavelength; only fluxes at $\lambda 5100 \AA$ in ergs cm ${ }^{-2} \mathrm{~s}^{-1} \AA^{-1}$ are available. The Structure Function presented in Fig. 6 (bottom panel) was calculated using all these data.

\subsection{Monte-Carlo simulations of the light curves of the NGC 5548 and their Structure Function}

Using the Structure Function technique, all results are derived on the assumption that the variability of AGNs can be represented as a random process of a flicker noise or a shot noise type. Therefore, it is possible to do some modeling of the observed light curve using a Monte-Carlo simulation. Such modeling will help us, for instance, to discard events due to the gaps in the data. This is a problem that calls for a separate consideration: a paper on the Structure Function technique with Monte-Carlo simulations of different kinds of light curves is now in preparation by Sergeev \& Merkulova (2001). In this section results of Monte-Carlo simulations of the Structure Function of NGC 5548 are presented.

The so-called shot noise represents the random process set by the sum of a large number of short-term pulses (flares). The shape of the flares should be constant, and the amplitude is a random variable with a constant dispersion. The sequence of the moments of flares reflects a Poisson process, i.e., the moments of flares are randomly scattered over time. Let data trains be a superposition of separate flares. We also assume a power law distribution of flares, both of duration and of amplitude. We assume that the amplitude of the flares decreases with decreasing duration. 

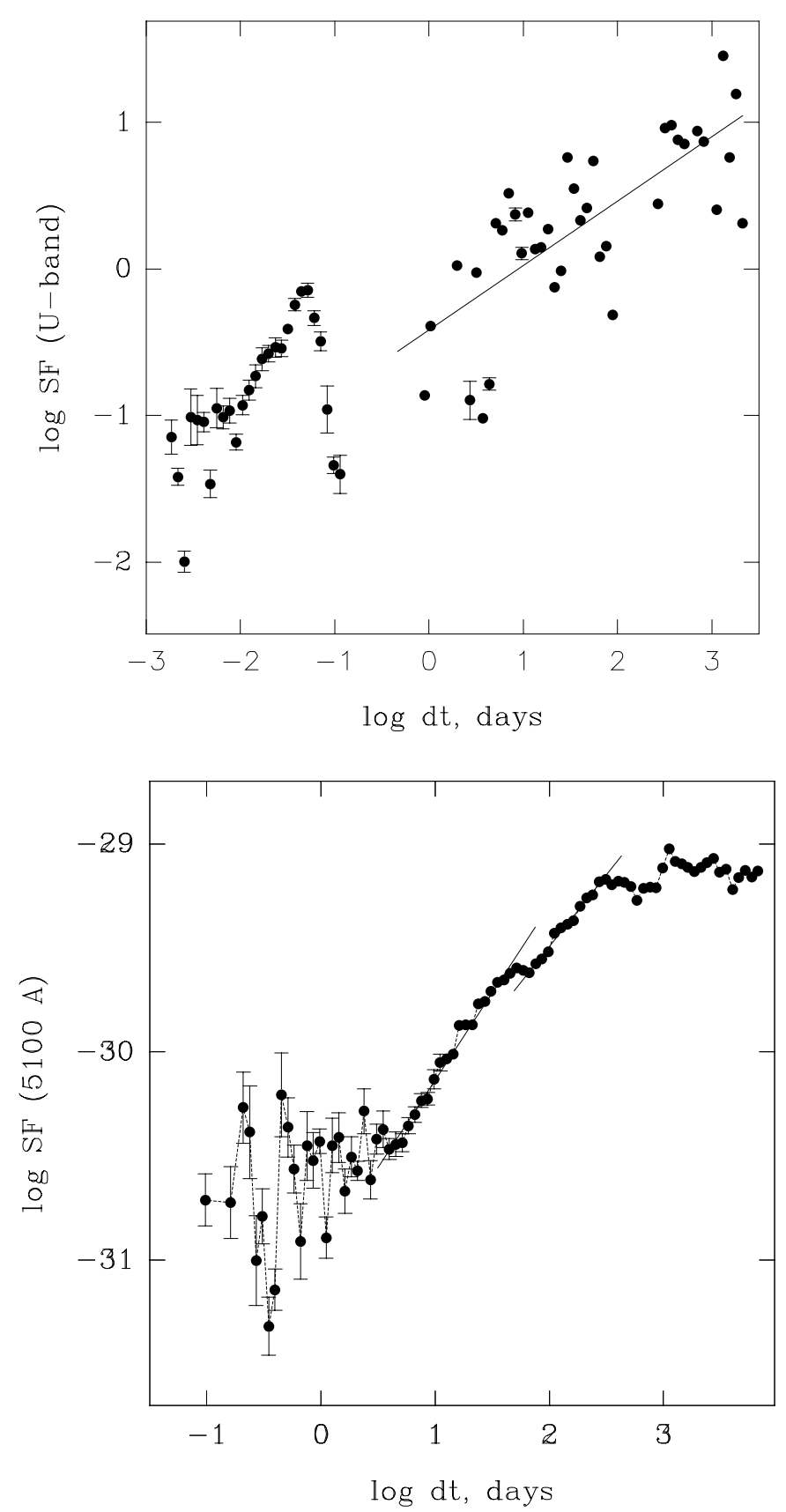

Fig. 6. Structure Functions of the variable flux of the NGC 5548 nucleus calculated from $U B V R I$ observations (for clarity, the top panel only shows the $S F$ for the $U$ band), and from the observations at $\lambda 5100 \AA$ obtained during AGN Watch campaigns and some others (bottom panel; see text). Error bars of $S F$ s are absent in cases when their dimensions are smaller than the circle. Straight lines are regression lines.

Data trains were created with the above-mentioned noise characteristics using flare parameters that do not contradict the observations of the Seyfert galaxies. The initial noise process was chosen to have a value of " $b$ " very similar to that calculated from the observed light curve.

The following model of flares was used for data-train modulation: a minimal duration of the flare of 0.001 day, and a maximal duration of the flare of 50 days, a mean

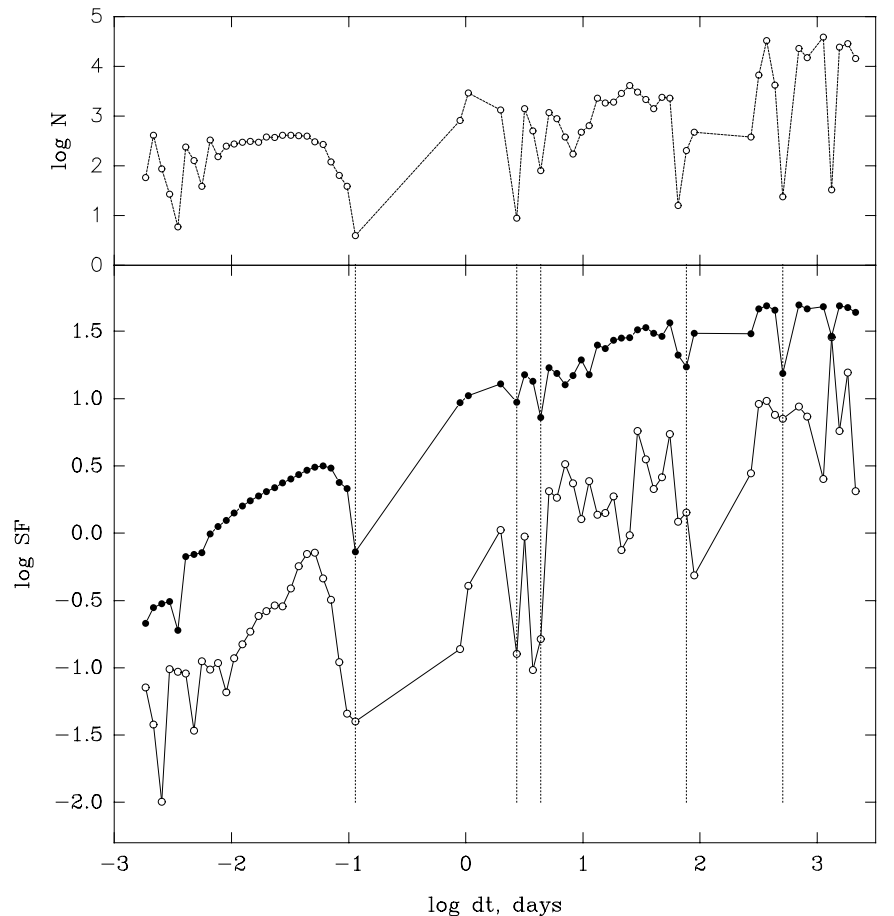

Fig. 7. Bottom panel: comparison of the Structure Function for observations of the NGC 5548 nucleus obtained in the $U-$ band (open circles) with the $S F$ for the simulated "light curve" calculated as a mean from 500 simulations (filled circles; see text). Error bars are random noise at a level 1\%. Vertical dotted lines indicate minima that are identical on both $S F$ s; they are due to the gaps in the data sampling. The plot in the top panel corroborates this conclusion; these are logarithms of the number of pairs $N$ falling within each bin against $\log \mathrm{d} t$.

duration between two successive flares equal to 0.05 day, i.e. one flare occurs per 1.2 hour. Let the dependence of the distribution of number of flares $n$ on their duration $\omega$ be $n(\omega) \sim \omega^{\alpha}$, and let the dependence of distribution of amplitude A on flare duration be $A(\omega) \sim \omega^{\beta}$. We assume $\alpha=-1.8$ and $\beta=0.07$, which yields " $b$ " $=0.35$ for the following range of time lag: 0.001 day $\lesssim$ lag $\lesssim 50$ days.

500 data trains were calculated using these noise characteristics, and all simulated "light curves" were sampled with the same patterns as the real data. Then random noise at a level of 1 percent was added to every simulated "light curve". Structure Functions were calculated using these 500 simulated "light curves", and slopes " $b$ " were evaluated. The mean slope " $b$ " calculated from these 500 $S F$ s and its standard deviation are equal to $0.355 \pm 0.007$. All 500 Structure Functions were averaged and the mean simulated $S F$ showed close agreement with the real one (see Fig. 7). This figure shows a comparison of the $S F$ obtained from the $U B V R I$ observations (bottom panel: open circles; $S F$ in the $U$-band) with the simulated $S F$ (bottom panel: filled circles). Vertical lines show minima that are identical on both $S F$, and that are due to the gaps in the data sampling. The top panel shows the logarithms of the number of pairs $N$ falling within a bin determined by the lag $\mathrm{d} t$ (also on a logarithmic scale). It is clear that 
Table 2. Structure function parameters of long-term variability of the NGC 5548 nucleus in 1993-1999, obtained from simultaneous $U B V R I$ observations.

\begin{tabular}{cccc}
\hline \hline $\begin{array}{c}\text { Spectral } \\
\text { region }\end{array}$ & $\begin{array}{c}\text { Time } \\
\text { interval }\end{array}$ & $b$ & $r$ \\
\hline 1 & 2 & 3 & 4 \\
\hline$U$ & $1 \mathrm{~d}-2100 \mathrm{~d}$ & $0.43 \pm 0.06$ & $0.74 \pm 0.05$ \\
$B$ & $-"-$ & $0.37 \pm 0.06$ & $0.71 \pm 0.05$ \\
$V$ & $-"-$ & $0.29 \pm 0.07$ & $0.58 \pm 0.06$ \\
$R$ & $-"-$ & $0.40 \pm 0.07$ & $0.67 \pm 0.06$ \\
$I$ & $-"-$ & $0.29 \pm 0.06$ & $0.65 \pm 0.06$ \\
\hline
\end{tabular}

the minima indicated by the vertical lines are in fact due to the gaps in the data.

It is clear that the above assumptions and parameters of noise process fit the real Structure Function well:

1) The $S F$ in the $U$-band (Fig. 7) may be represented by a regression line with slope: $b=0.353 \pm 0.026$ (from the first to the final point); and by a regression line for the intranight part with slope $b_{1}=0.887 \pm 0.106$.

2) The mean simulated $S F$ in Fig. 7 may be analogously represented by regression lines with slopes $b_{\text {sim }}=$ $0.355 \pm 0.015$ (from the first to the final point) and $b_{1, \operatorname{sim}}=0.859 \pm 0.016$ (for the intranight part).

\subsection{Variability characteristics of NGC 5548 obtained from SFs for long-term variability ( $d t \geq 1$ day)}

Parameters of the Structure Functions are presented in Table 2 for long-term variability in the $U B V R I$ bands, for the whole observing time. Column 1 gives the wave bands, time intervals for possible correlated variability are specified in Col. 2, slopes "b" are shown in Col. 3, correlation coefficients for the corresponding regression lines are given in Col. 4.

One can see that the processes of mixed flicker-noise and shot-noise types are observed in different wave bands, and with different slopes.

From Fig. 6 (bottom panel; observations at $\lambda 5100 \AA$ ), a variability on a time scale of from days to hundreds of days is characterized by flares with different slopes and different durations. All needed information is shown in Table 3 as follows: Col. 1: spectral region; Col. 2: time intervals for the correlated variability shown in Fig. 6 (bottom panel); Col. 3: values of $T_{\max }$ characterizing the flares duration; slopes " $b$ " and correlation coefficients for regression lines in Fig. 6 are in Cols. 4 and 5, respectively. All parameters are shown with their rms errors. It is clear that well-sampled data make it possible to derive from the light curve some flares with durations of $14,52,98,163$, and 310 days.

Processes causing long-term variability observed during analogous observations of other galaxies from Table 1 are compared in Fig. 8. From this figure, all Seyfert 1 galaxies show processes of a similar type (mixed shot noise and flicker noise). The BL Lac object NGC 1275, is distinguished from these Sy1 galaxies not only by its pro-

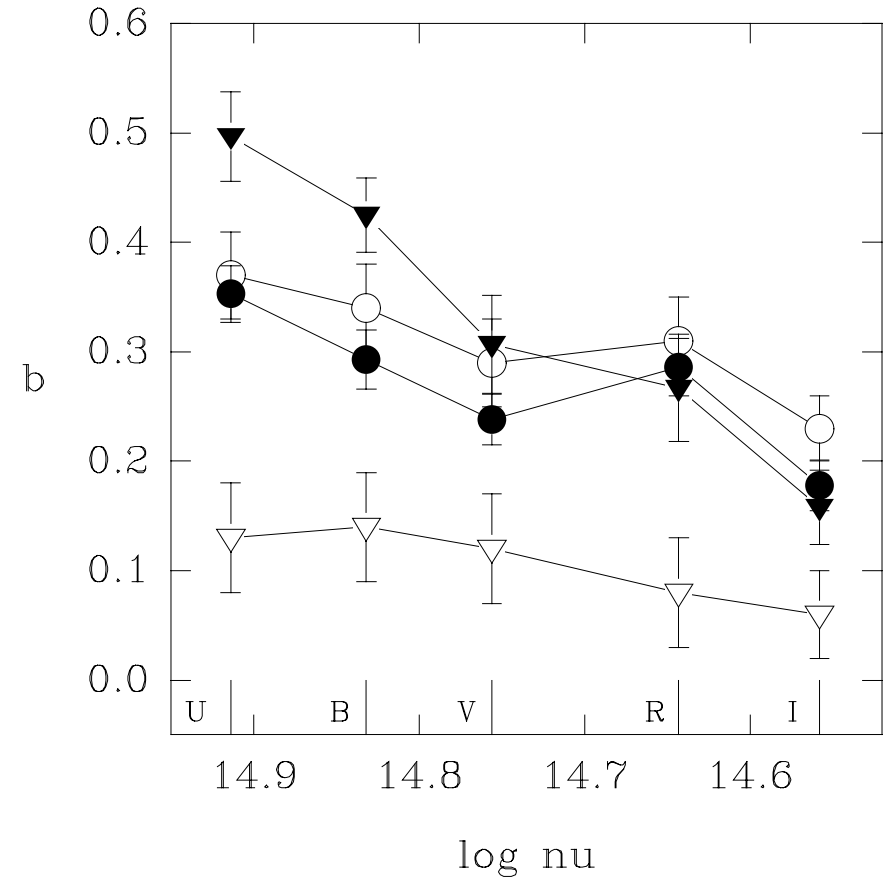

Fig. 8. Frequency dependencies of slopes " $b$ " for 5 spectral bands obtained from Structure Functions calculated for long-term variability of 4 galaxies: NGC 5548 (filled circles), NGC 7469 (open circles), NGC 4151 (filled triangles), and NGC 1275 (open triangles). Vertical bars are error bars obtained while $S F$ calculations. The locations of $U B V R I$ wave bands are given by vertical lines in the abscissa.

Table 3. Structure function parameters of long-term variability of the NGC 5548 nucleus, obtained using data of International AGN Watch campaigns in 1993-1999 (Peterson et al. 1999) and a sample of "Pre-AGN Watch" (Sergeev et al. 1997).

\begin{tabular}{ccccc}
\hline \hline $\begin{array}{c}\text { Spectral } \\
\text { region }\end{array}$ & $\begin{array}{c}\text { Time } \\
\text { interval }\end{array}$ & $T_{\max }$ & $b$ & $r$ \\
\hline 1 & 2 & 3 & 4 & 5 \\
\hline $5100 \AA$ & $4 \mathrm{~d}-52 \mathrm{~d}$ & $14 \mathrm{~d}, 52 \mathrm{~d}$ & $0.85 \pm 0.03$ & $0.99 \pm 0.01$ \\
$5100 \AA$ & $67 \mathrm{~d}-310 \mathrm{~d}$ & $98 \mathrm{~d}, 163 \mathrm{~d}$ & $0.69 \pm 0.02$ & $0.99 \pm 0.01$ \\
\hline
\end{tabular}

cess type (almost pure flicker-noise), but also by the slope, which is approximately equal for all bands, indicating that identical types of processes occur in all wave bands on long timescales.

\subsection{SFs for intranight variations}

Structure Functions for intranight variability of NGC 5548 in $U B V R I$ bands are shown in Fig. 9. From this figure it can be seen that intranight variability in different bands is characterized by different slopes and different values of $T_{\max }$, i.e. flares with different durations are present. Therefore, regression lines on $S F$ s are shown for time intervals where slopes " $b$ " are well defined. Two types of flare are derived from these plots for different time intervals 

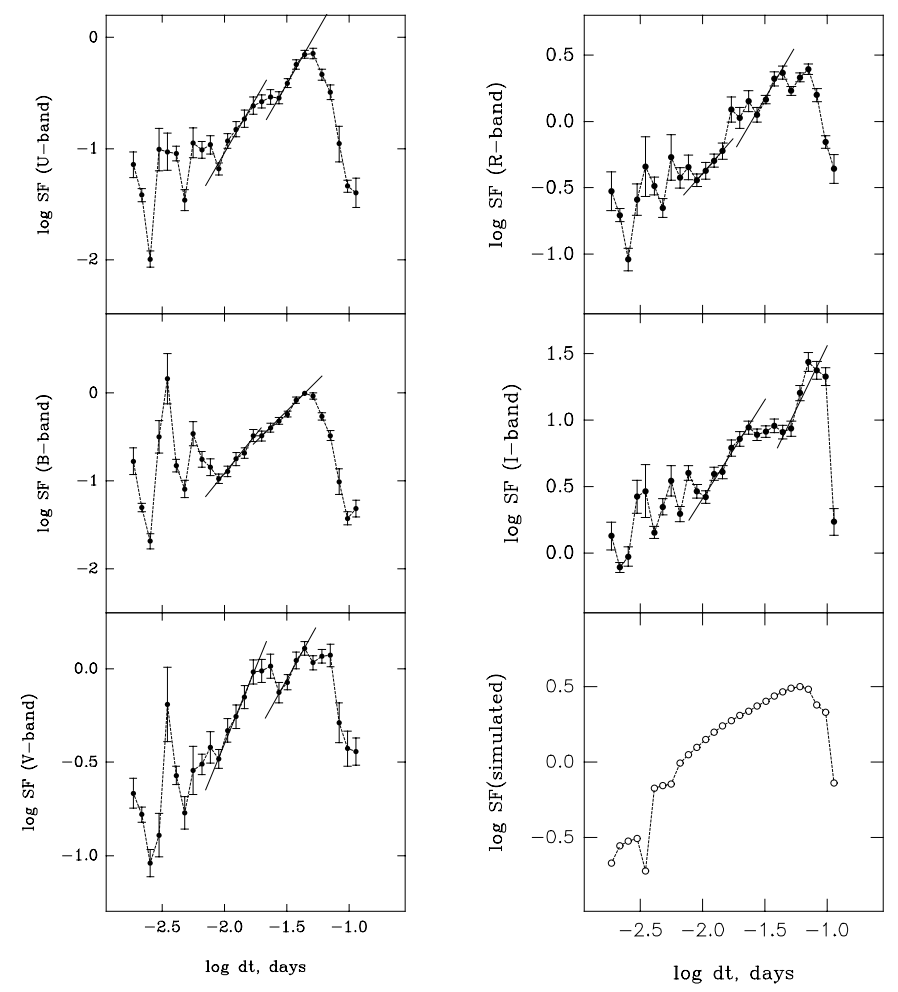

Fig. 9. Structure Functions of the nucleus of NGC 5548 in $U B V R I$ bands for intranight variations. Vertical bars are error bars obtained in the $S F$ calculations. Regression lines are indicated for two different time intervals, where a good correlation was found (see text). The simulated $S F$ for intranight variations is plotted on the right bottom panel (open circles). No steps are seen in this $S F$.

which are indicated in this figure by two different lines. The intranight part of the simulated SF (see Sect. 4.1) is plotted on the right bottom panel. As the model does not allow us to create $S F$ s with two slopes on their intranight parts at a confidence level of $98 \%$, we can conclude that the two type of flares derived in $S F$ s are real.

The parameters of these $S F$ s for intranight variations of NGC 5548 are presented in Table 4, as follows: Col. (1) - spectral band, Cols. (2) and (5) - time intervals for correlated variability, Cols. (3) and (6) - slopes " $b$ " with rms, Cols. (4) and (7) - correlation coefficients and their rms.

Two types of flares are present in the intranight light curves of the NGC 5548 nucleus (see Table 4): the more rapid ones (durations of 21-34 min) are better seen in the UV region, where slopes " $b$ " are equal to 1.94-1.61; those less rapid (durations of $63-102 \mathrm{~min}$ ) are clearly present both in the $U$ band $(b=1.94)$ and in the $R, I$ bands $(b=$ 1.61 and 1.99). It is clear that the slopes of the intranight variations of the NGC 5548 nuclear region depend on the spectral band.

The frequency dependencies of slopes $b$ for the four galaxies in question are shown in Fig. 10. Two types of flares are observed in the light curves of the nucleus of each galaxy; they are shown by open and filled symbols: if flares are more pronounced in the UV region, they are shown by open circles (NGC 5548), triangles down (NGC 7469), squares (NGC 4151), or triangles up (NGC 1275); in contrast, if the flares are more pronounced in the red, that is indicated by corresponding filled symbols. From Fig. 10 it can be seen that the nucleus of NGC 5548 shows two above-mentioned flares of shot-noise type in the intranight light curves (open and filled circles, respectively).

Two levels of microvariability were revealed in intranight light curves of NGC 1275 by Pronik et al. (1999b): high-level variability with $T_{\max }=4 \mathrm{hr}$ is caused by almost pure shot- noise processes, with the highest slope in the $U$ band (open triangles up in Fig. 10); low-level variability with $T_{\max }=56 \mathrm{~min}$ is caused by a mixed flicker-noise and shot-noise process, showing up most clearly in the $R$, $I$ bands (filled triangles up in Fig. 10).

In intranight light curves of NGC 7469 there are two types of variability present with different slopes (open and filled triangles down in Fig. 10). The results on the analysis of the intranight variability of NGC 7469 have been published by Merkulova (2000).

NGC 4151 shows intranight processes of a shot-noise type, too, but they are almost identical in all wave bands and have approximately equal slopes (open and filled squares) (Merkulova et al. 2001).

\subsection{Comparison with other sources}

From the Structure Function analysis of the data samples of the nucleus of NGC 5548, NGC 4151, NGC 7469, and NGC 1275 the following conclusions can be made:

1) all galaxies show a mixture of flicker noise and shot noise on a time scale of years. NGC 4151, NGC 5548 and NGC 7469 show similar slopes at all wave bands, excluding the UV region, where the contribution of shot noise is essentially higher for NGC 4151, but this parameter is less sensitive to the amplitude of a new cycle of activity than parameter $R_{\max }$ (Fig. 5). NGC 1275 shows almost equal slopes " $b$ " (similar to $R_{\max }$ in Fig. 5), indicating that the flicker-noise process operates in all wave bands.

2 ) slopes " $b$ " for intranight variability on a time scale of hours indicated that the contribution of the shot-noise process is essential, particularly for NGC 5548. It is interesting that almost equal slopes " $b$ " are observed in $S F$ s of NGC 4151, indicating similar processes in all spectral bands.

3) two types of flares were deduced from the analysis of intranight variations of the four galaxies, and different slopes " $b$ " characterizing intranight light curves show different wavelength dependencies for different galaxies, indicating that different sources are probably working in the nuclear regions of NGC 5548, NGC 7469 and NGC 1275, but in the case of NGC 4151 only the one source is responsible for intranight variations.

\section{Statistical parameters of intranight variability}

In order to examine the intranight variations of the nucleus of NGC 5548, and following the paper by GonzálezPérez et al. (1996), the values for the standard deviations 
Table 4. Structure function parameters of intranight variability of the NGC 5548 nucleus in $U B V R I$ bands in $1993-1999$.

\begin{tabular}{ccccccc}
\hline $\begin{array}{c}\text { Spectral } \\
\text { region }\end{array}$ & $\begin{array}{c}\text { Time } \\
\text { interval }\end{array}$ & $b$ & $r$ & $\begin{array}{c}\text { Time } \\
\text { interval }\end{array}$ & $b$ & $r$ \\
\hline 1 & 2 & 3 & 4 & 5 & 6 & 7 \\
\hline$U$ & $13 \mathrm{~m}-24 \mathrm{~m}$ & $1.94 \pm 0.25$ & $0.98 \pm 0.02$ & $39 \mathrm{~m}-63 \mathrm{~m}$ & $1.94 \pm 0.15$ & $0.99 \pm 0.01$ \\
$B$ & $13 \mathrm{~m}-24 \mathrm{~m}$ & $1.73 \pm 0.17$ & $0.99 \pm 0.14$ & $29 \mathrm{~m}-63 \mathrm{~m}$ & $1.42 \pm 0.08$ & $0.99 \pm 0.02$ \\
$V$ & $13 \mathrm{~m}-24 \mathrm{~m}$ & $1.61 \pm 0.10$ & $0.99 \pm 0.01$ & $39 \mathrm{~m}-63 \mathrm{~m}$ & $1.20 \pm 0.12$ & $0.99 \pm 0.01$ \\
$R$ & $13 \mathrm{~m}-21 \mathrm{~m}$ & $1.07 \pm 0.01$ & $1.00 \pm 0.00$ & $39 \mathrm{~m}-63 \mathrm{~m}$ & $1.61 \pm 0.22$ & $0.98 \pm 0.02$ \\
$I$ & $15 \mathrm{~m}-34 \mathrm{~m}$ & $1.49 \pm 0.14$ & $0.98 \pm 0.01$ & $63 \mathrm{~m}-102 \mathrm{~m}$ & $1.99 \pm 0.55$ & $0.96 \pm 0.04$ \\
\hline
\end{tabular}

Table 5. Variability parameters $S D / F(\%)$ at $U, B, V, R$ and $I$ bands of intranight light curves of NGC 5548 , represented in Fig. 11.

\begin{tabular}{ccccccccc}
\hline \hline Date & JD & $n$ & seeing & $S D / F(U)$ & $S D / F(B)$ & $S D / F(V)$ & $S D / F(R)$ & $S D / F(I)$ \\
\hline 1 & 2 & 3 & 4 & 5 & 6 & 7 & 8 & 9 \\
\hline 01/02 May 1995 & 2449839 & 24 & $2^{\prime \prime} \cdot 0$ & 7.7 & 7.7 & 2.6 & 1.1 & 2.6 \\
22/23 June 1998 & 2450987 & 18 & $2 . .5$ & 7.3 & 6.7 & 7.9 & 7.1 & 9.3 \\
28/29 June 1998 & 2450993 & 28 & $2^{\prime \prime} \cdot 0$ & 1.0 & 0.9 & 1.0 & 0.6 & 1.7 \\
\hline
\end{tabular}

$(S D)$ of the nightly averaged flux $F$, and of $S D / F$, which is a measure of the intranight variability, were calculated for each night of the observations. The ratio $S D / F$ is preferable as a measure of small variations, as it is calculated statistically. This parameter is a measure of the variability amplitude over a night, which is independent of the galaxy's brightness. Values of $S D / F$ are independent of seeing; for example, if $S D / F$ is plotted versus seeing, the regression coefficients are equal to 0.148 for the $U$ band, and 0.042 for the $I$ band, respectively. Most observations were made during the nights with seeing $\leq 3^{\prime \prime}$.

Representative light curves displaying the observed microvariability in five spectral bands $U B V R I$ (bottom to top in each panel, respectively) obtained over three nights are given in Fig. 11. Vertical axes give the brightness of the nucleus in $\mathrm{mJy}$, the vertical bars indicate the rms errors about the mean obtained by averaging 8 integrations.

Table 5 lists the variability parameters $\mathrm{S} D / F(\%)$ for these nights (Cols. 5-9), together with dates and Julian Dates (Cols. 1, 2), number of measurements $n$ (Col. 3), and seeing during the night in $\operatorname{arcsec}(\mathrm{Col} .4)$.

In Fig. 11b (JD 245987) one can see a rapid flare obtained during a ground-based optical monitoring campaign on NGC 5548 in June 1998. The broad-band flux increased by $20 \pm 2 \%$ within $\approx 30$ min and decreased within $\approx 60$ min to the flux level at the beginning of this event. It was shown that this flux increase could not be due to temporal variations of a standard star (see Fig. 2 in Dietrich et al. 2001). This short continuum flare is described in detail in the cited paper.

For OJ 287 (a BL Lac type object) González-Pérez et al. (1996) found a moderately strong anti-correlation in the sense of the fainter the object, the greater the microvariability amplitude. One would expect such a cor- relation if all microvariability were generated by the same mechanism and typically involved the same level of energy generation (e.g. flickering on the accretion disc with a constant energy output), independent of background level. In Fig. 12, there is hardly any correlation of flux with intranight-variability amplitude; the correlation coefficients are: $r_{U}=0.174, r_{B}=0.067, r_{V}=0.146$, $r_{R}=0.138, r_{I}=0.086$. No correlation was found in the case of NGC 7469 (Merkulova 2000) either. Thus, the model of Disc Instability (DI) does not appear to be supported by the occurence of intranight variability.

It is interesting to have a look at the behavior of the amplitude of intranight variability (expressed in $S D / F$ ) for the whole observational sample. For clarity, only data in the $U$ and $I$ bands are shown in Fig. 13.

Table 6 contains the mean amplitudes of the intranight variability of NGC 5548 in each of the $U B V R I$ bands during the whole observing period (44 nights), as follows: Col. 1: spectral band, Col. 2: $S D / F$ averaged over 44 nights together with their rms errors. If we remove two points of greatest amplitude in the $U$ band (Fig. 13), the remaining data show a mean $S D / F=3.03 \pm 0.37 \%$. Similarly, when we remove one point corresponding to flaring activity in the $I$ band, the remaining data show a mean amplitude $S D / F=3.20 \pm 0.31 \%$. One can conclude, that the amplitude of intranight variability in the $U, B, V, I$ bands is equal to $\sim 3.36 \%$ (i.e. averaging the values in Table 6 ), and $\sim 2 \%$ in the $R$ band, where the stellar contamination is greatest, decreasing the level of intranight variability at this band.

Intranight variability does not manifest itself every night, therefore the mean amplitude does not characterize microvariability fully. A probability characteristic is needed. To this end the ratios $P=N_{i} / N_{\text {tot }}$ of number 


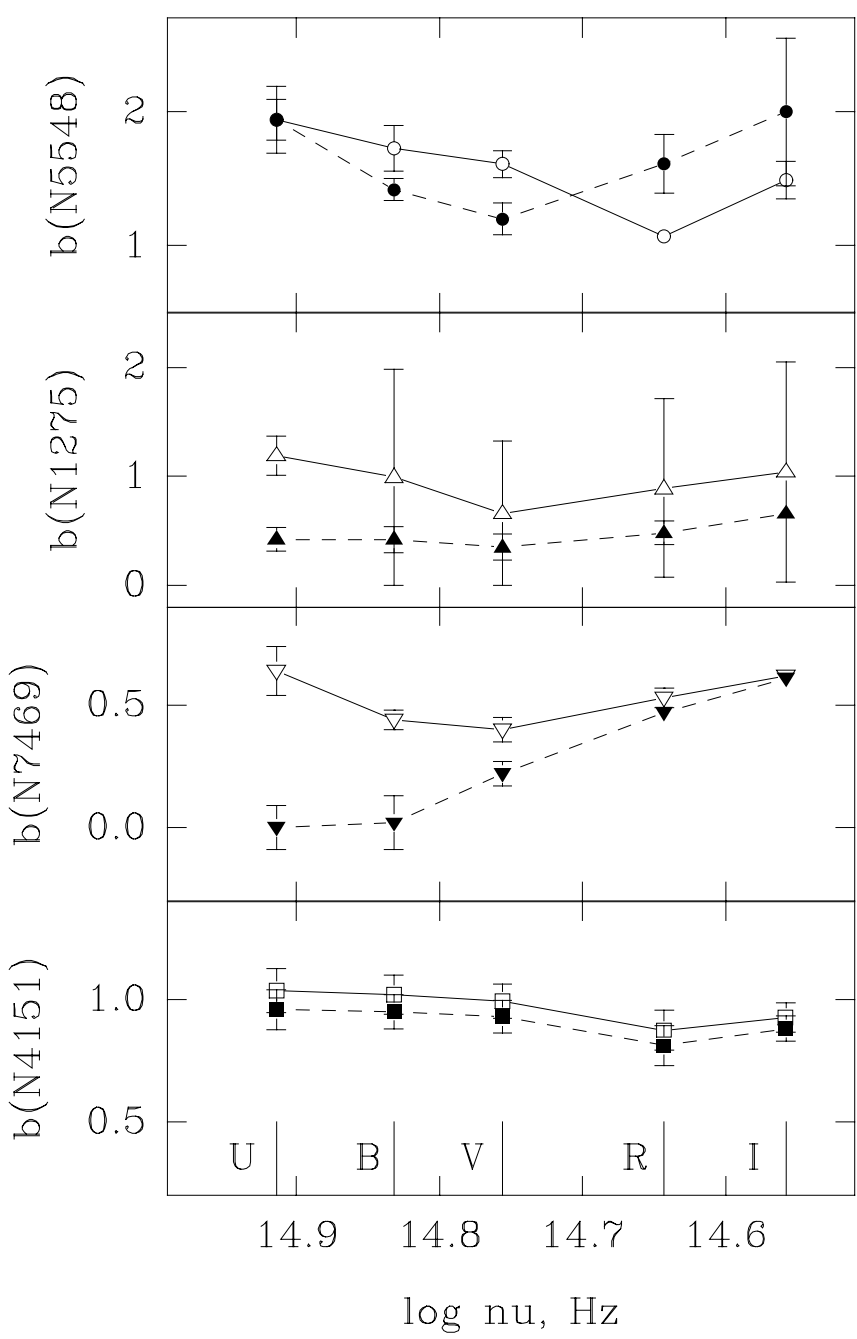

Fig. 10. Slopes " $b$ " for 5 spectral bands obtained from Structure Functions for intranight variations of 4 galaxies: NGC 5548 (open and filled circles); NGC 1275 (open and filled triangles up); NGC 7469 (open and filled triangles down); and NGC 4151 (open and filled squares). Open and filled symbols corresponding to two types of flares have been extracted from these $S F$ s: if flares are more pronounced in the UV region, their slopes " $b$ " are shown by open symbols and solid lines; flares more pronounced in the red are shown by filled symbols and dotted lines (see text). Vertical bars are error bars obtained from the $S F$ calculations. The locations of $U B V R I$ wave bands are given by vertical lines in the abscissa.

of nights with $S D / F \geq 1 \%, \geq 2 \%, \geq 3 \%$, and so forth, $\left(N_{i}\right)$, to all 44 observational nights, $\left(N_{\text {tot }}\right)$, were taken to represent the percentage of the probability of detecting variability at a given level, these ratios were plotted against the appropriate parameters $S D / F$ in Fig. 14 . The data in the $U, B, V, I$ bands coincided well, so they were averaged, while the data in the $R$-band differ from the others. These curves were fitted well by second-order exponential decay functions (solid lines in Fig. 14). From this figure, one can deduce, for example, that the probability of detecting intranight variability with an amplitude $\geq 2 \%$ in the $U, B, V, I$ bands is equal to $\sim 55 \%$, while in the $R$ band this probability is $\sim 40 \%$. Some nights with excessive
Table 6. Mean amplitudes of intranight variability of the NGC 5548 nucleus in 1993-1999.

\begin{tabular}{cc}
\hline \hline $\begin{array}{c}\text { Spectral } \\
\text { region }\end{array}$ & $\begin{array}{c}S D / F \\
\% \%\end{array}$ \\
\hline 1 & 2 \\
\hline$U$ & $3.75 \pm 0.62$ \\
$B$ & $3.27 \pm 0.44$ \\
$V$ & $2.98 \pm 0.37$ \\
$R$ & $1.97 \pm 0.20$ \\
$I$ & $3.43 \pm 0.38$ \\
\hline
\end{tabular}

variability (amplitudes in the range $5-7 \%$ ) are marked by open triangles in Fig. 14.

As has been shown in this paper, intranight variability may be a result of random processes. From this it follows that exponential functions should give good approximations of the probability curves, because random processes are fitted well with exponential and Gaussian functions. Thus, probability curves also demonstrate the random character of the short-term variability of Seyfert galaxies.

Analogous curves for the galaxies NGC 4151, NGC 7469 and NGC 1275 can be found in Figs. 8-10 in Merkulova (2000). These curves were fitted well by second-order exponential decay functions, too. To compare such curves for all galaxies discussed here, they are plotted in Fig. 15 by different symbols as indicated in the caption. The curves for each galaxy were averaged over all wave bands; bars are rms variations about these averages. Open triangles show the probability curve for data obtained in the $R$ band of NGC 5548. It differs essentially from the other curves, defining the lowest level of variability in the $R$ band. Averaging over the other four bands yields a curve that is near the probability curve of NGC 1275 (a BL Lac object), defining a level of variability, that is higher than those for the two other Sy1 galaxies, NGC 4151 and NGC 7469. Probability curves for these two galaxies coincided well. The mean brightness of NGC 1275 and NGC 5548 is less than that of NGC 4151 and NGC 7469, indicating that the difference in Fig. 15 might be caused by observational errors. However, the separate location of the probability curve for the $R$ band of NGC 5548 contradicts this supposition. As a result, one can conclude that intranight variability is really transient in character and manifests itself with different probabilities for different galaxies.

Assuming that the intranight variability can be detected at a level as low as $S D / F \geq 1 \%$, the probability of detecting variability on such a level could be translated into a duty cycle (the fraction of time when the galaxy is variable), characterizing the efficiency of the central energy source. Duty cycles for NGC 5548, NGC 4151, NGC 7469 and NGC 1275 are shown in Fig. 16 for variability level $S D / F \geq 1 \%$. In general, duty cycles for all 


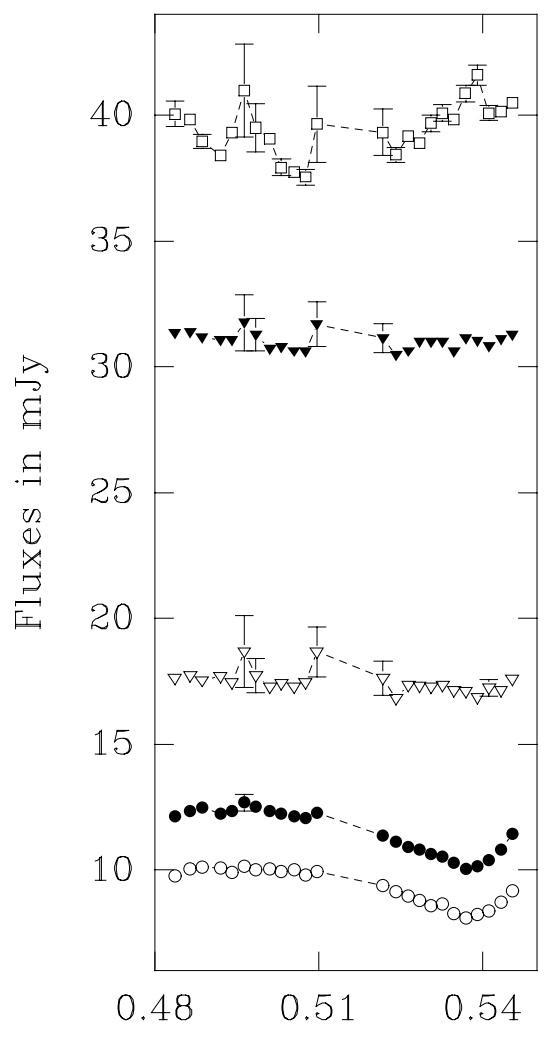

JD2449839+
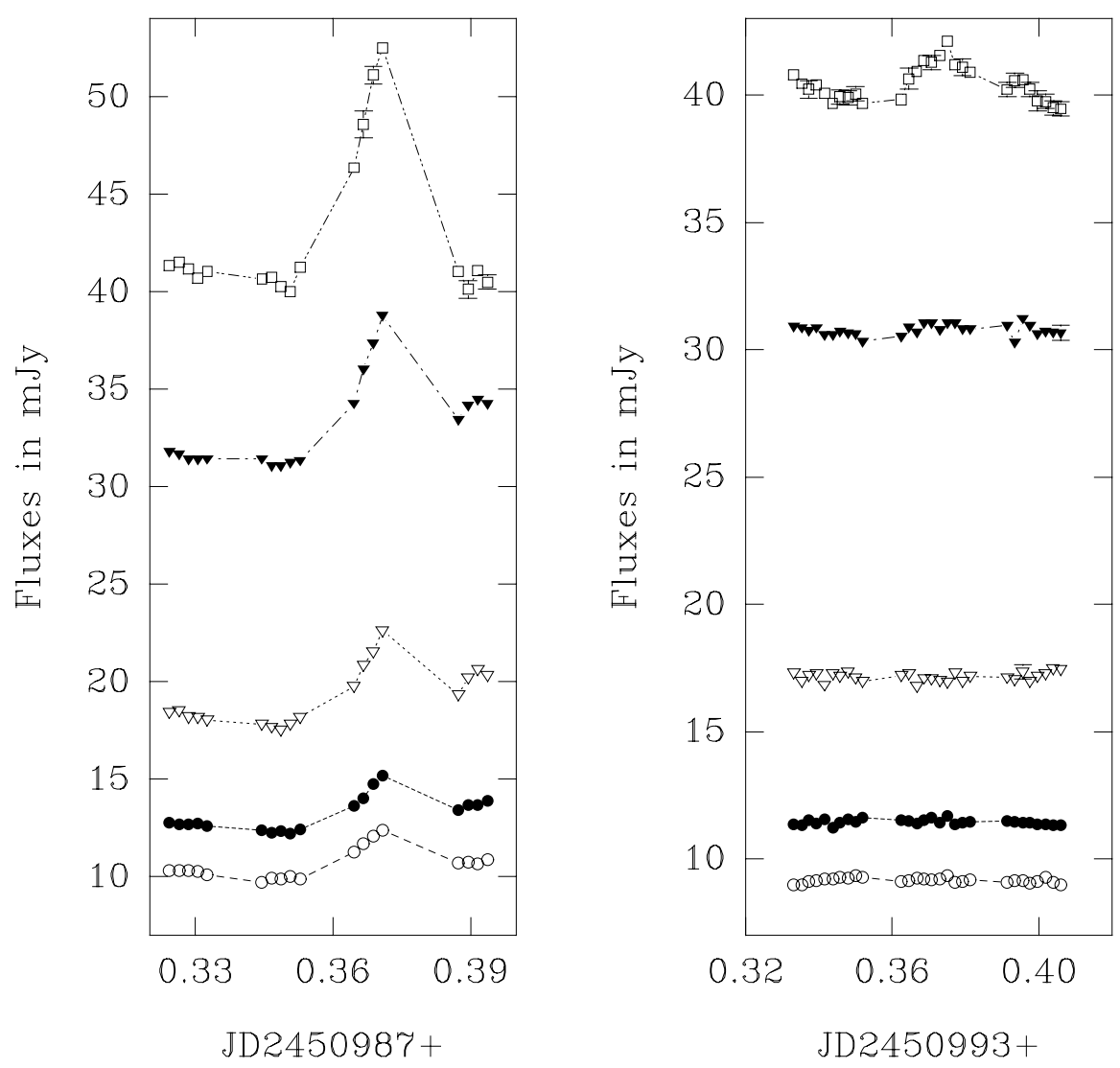

Fig. 11. Intranight light curves: a) JD 2449839; b) JD 2450987; c) JD 2450993 (see Table 5 for more information).

galaxies are greater than 0.5 . Variability independent on wavelength is detected in the nuclear region of NGC 4151. In the case of NGC 5548 and NGC 7469 the efficiency of their central sources is lower in the $R$ band, probably because of the influence of stellar contamination. The independent IR source in NGC 7469 may cause the high duty cycle in the $I$ band (Merkulova 2000).

\section{Results and discussion}

\subsection{Results}

Observations of the nuclear region of the Seyfert galaxy NGC 5548 obtained at the Crimean Astrophysical Observatory with the $1.25 \mathrm{~m}$ telescope are used to examine the continuum variability. Over 44 nights comprising 7 observational runs between 1993 March 22 and 1999 July 10 in each spectral band of Johnson's UBVRI system 672 measurements have been performed simultaneously through a circular aperture $15^{\prime \prime}$ in diameter. Observations of the nucleus of NGC 5548 were compared with analogous data samples of other Sy galaxies: NGC 4151 (Merkulova et al. 1999), NGC 1275 (Pronik et al. 1999a), and NGC 7469 (Merkulova 2000). The homogeneity of data obtained with the same telescope, using the same technique has made statistical comparison possible among different galaxies and different wave bands.
The following conclusions are reached from the analysis of the data:

1) A peak amplitude $R_{\max }=F_{\max } / F_{\min }=2.99$ for the whole light curve of the NGC 5548 nucleus was observed in the $U$ band, while a minimum amplitude $F_{\max } / F_{\min }=1.37$ was observed in the $I$ band during the whole observation period. From a comparison with other Seyfert galaxies discussed here, it is concluded that the amplitude of long-term variations is higher in the UV region than in the near IR, where stellar contamination is higher. Interestingly, for NGC 1275 shows the amplitude of the long-term variability is practically independent of wave band.

2) Variability on a time scale of from days to hundreds of days is characterized by flares with different slopes and different durations. Well-sampled data of the International AGN Watch campaigns make it possible to define flares with durations of 14, 52, 98, 163, and 310 days, using $S F$ s.

3) Data samples of $U B V R I$ observations of NGC 5548, were used to calculate Structure Functions; the $S F$ parameters were compared with those calculated for other galaxies discussed. The following conclusions are drawn:

a) All galaxies show a mixture of flicker noise and shot noise on a time scale of years. NGC 4151, NGC 5548 and NGC 7469 show similar slopes in all wave bands, excluding the UV region, where the contribution of shot noise is essentially higher for NGC 4151; the slope is less sensitive for the amplitude of a new cycle of activity than the 

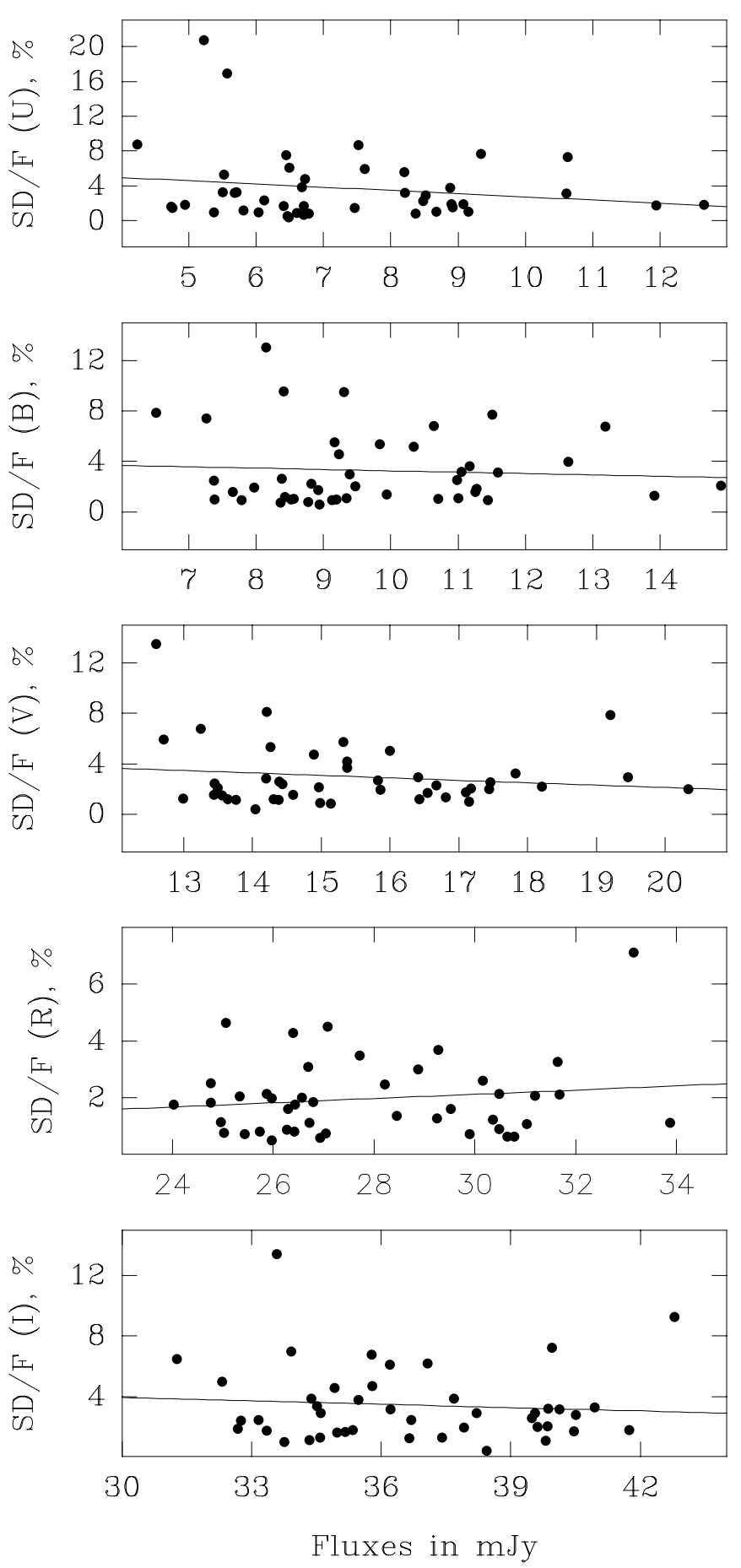

Fig. 12. $S D / F$ - flux density for intranight variations of the NGC 5548 nucleus in 1993-1999. The straight lines are regression lines.

parameter $R_{\max }$. NGC 1275 shows almost equal slopes " $b$ " (similar to the parameter $R_{\max }$ ), indicating that flicker noise is dominant in all wave bands.

b) Slopes " $b$ " for intranight variability on a time scale of hours indicate that shot noise is dominant, particularly for NGC 5548. It is interesting, that almost equal slopes " $b$ " are observed in the $S F$ s of NGC 4151, indicating that on this time scale shot noise dominates in all spectral bands.
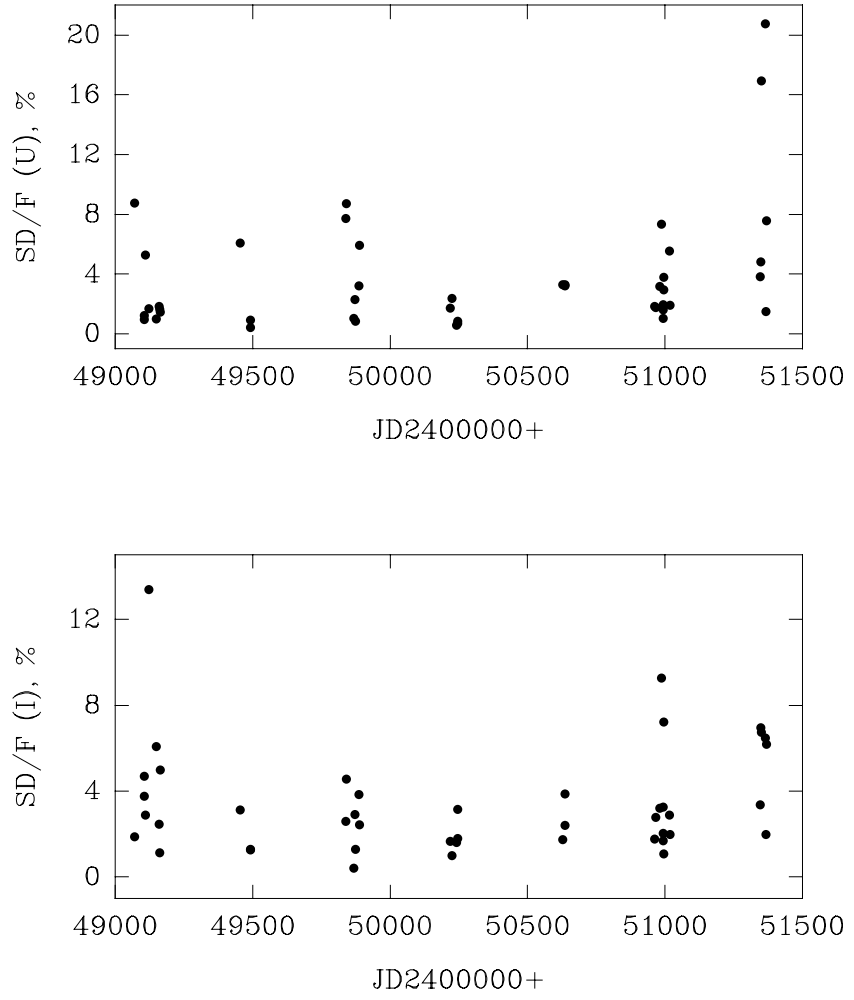

Fig. 13. Behavior of the amplitude of intranight variability (in $S D / F$ ) for the whole observational sample (44 Julian Dates). For clarity, only data in the $U$ and $I$ bands are shown.

c) Two types of rapid flares occur in the $S F$ s of the NGC 5548 nucleus: the more rapid ones (with durations of 21-34 min) are more distinct in the UV region; those less rapid (with durations of 63-102 min) are equally strongly present in the $U$ band as in the $R, I$ bands. It is clear, that the slopes of intranight variations depend on the spectral band.

d) Two types of flares were derived from the analysis of intranight variations of the four galaxies in question, and different slopes " $b$ " characterizing the intranight light curves show different wavelength dependencies for different galaxies, indicating that more than one source of variability is at work in the nuclear regions of NGC 5548, NGC 7469 and NGC 1275, but in the case of NGC 4151 only one source is responsible for intranight variations.

4) In order to examine the intranight variations of the nucleus of NGC 5548, standard deviations $(S D)$ of the nightly averaged flux $F$, and a measure of intranight variability $S D / F$, were calculated for each night. This parameter is equivalent to the amplitude of variability over a night, and it is independent of the galaxy's brightness.

5) The amplitude of intranight variability of the nucleus of NGC 5548 in $S D / F$ units in the $U B V I$ bands is equal to $\sim 3.36 \%$ when averaged, and $\sim 2 \%$ in the $R$-band, where the stellar contamination is greatest, decreasing the level of intranight variability in this band.

6) Intranight variability does not manifest itself every night, therefore, the mean amplitude does not characterize microvariability fully. A probability characteristics is 


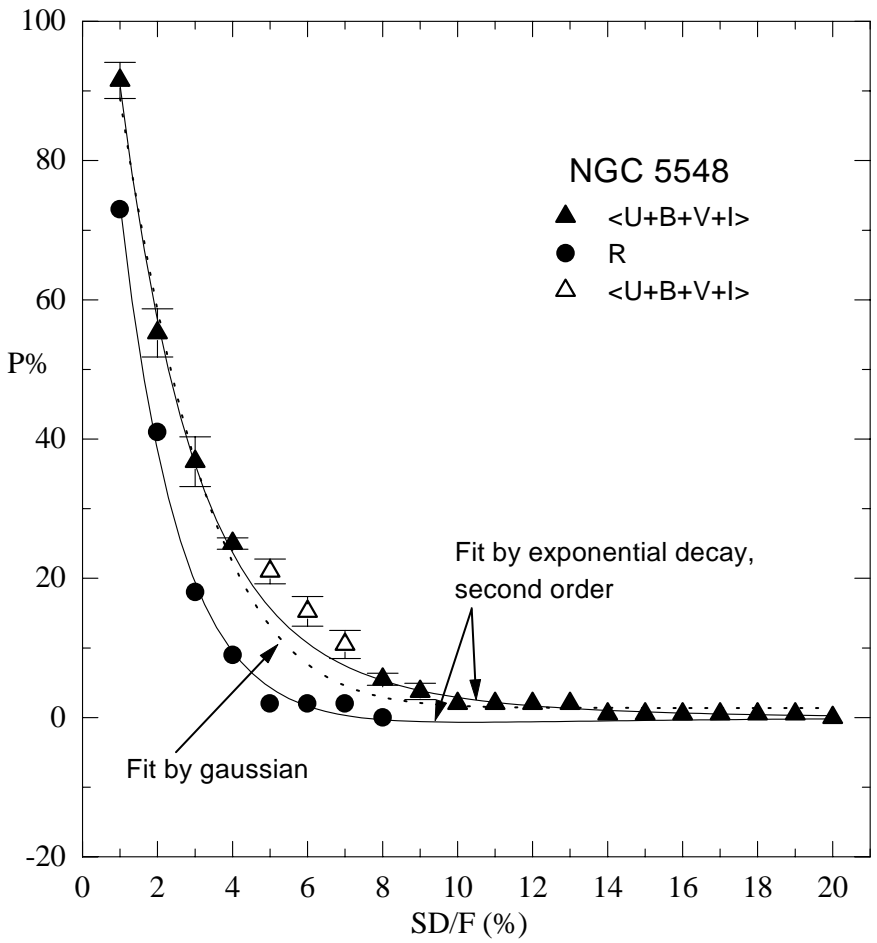

Fig. 14. A "probability curve" for NGC 5548: The ratios $P=$ $N_{i} / N_{\text {tot }}$ of the number of nights with $S D / F \geq 1 \%, \geq 2 \%, \geq 3 \%$, and so forth, $\left(N_{i}\right)$, to total number of nights, $\left(N_{\text {tot }}\right)$, expressed as a percentage of probability of detecting variability at a given level versus the matching $S D / F$. Probabilities averaged for the $U B V I$ wave bands are shown by triangles, with error bars indicating their rms variations. The data for the $R$ band are given as filled circles. An excess of nights with amplitudes in the range $5-8 \%$ is shown by open triangles. The dotted line is a Gaussian fit through the filled triangles. Solid lines are fits by exponential decay, second order. See text.

introduced as the percentage of probability of detecting intranight variability at a given level, resulting in a "probability curve". For example, in the case of NGC 5548, the probability of detecting intranight variability with an amplitude $\geq 2 \%$ in the $U, B, V, I$ bands is equal to $\sim 55 \%$, while in the $R$ band it is equal to $\sim 40 \%$. There are even some nights on which amplitudes in the range $5-7 \%$ are observed.

7) Assuming that the intranight variability can be detected for $S D / F$ as low as $1 \%$, the probability of detecting variability on such a level could be translated into a duty cycle (this is the fraction of time, where the galaxy is variable), characterizing the efficiency of the central energy source. Duty cycles for NGC 5548, NGC 4151, NGC 7469 and NGC 1275 are more than 0.5. Variability with the same amplitudes in all wave bands is detected in the nuclear region of NGC 4151. In the case of NGC 5548 and NGC 7469 the efficiencies of their central sources are lower in the $R$ band, probably because of the influence of the stellar contamination. The independent IR source in the NGC 7469 may cause high duty cycle values in the $I$ band (Merkulova 2000).

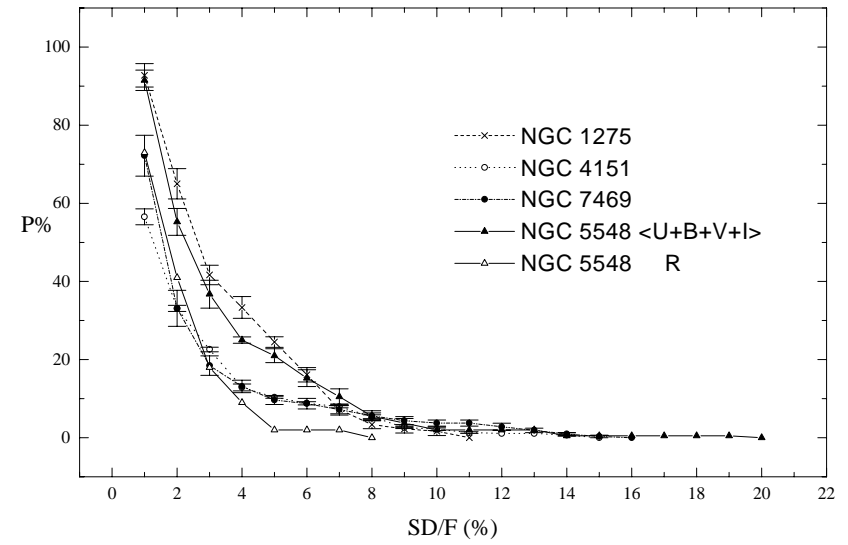

Fig. 15. The same curves as in Fig. 14, but for different galaxies: crosses: NGC 1275; open circles: NGC 4151; filled circles: NGC 7469. All these curves are averaged over 5 bands. The probability curves of NGC 5548 are averaged over the $U B V I$ bands (filled triangles); the same curve for the $R$ wave band is shown by open triangles. Vertical bars are error bars.

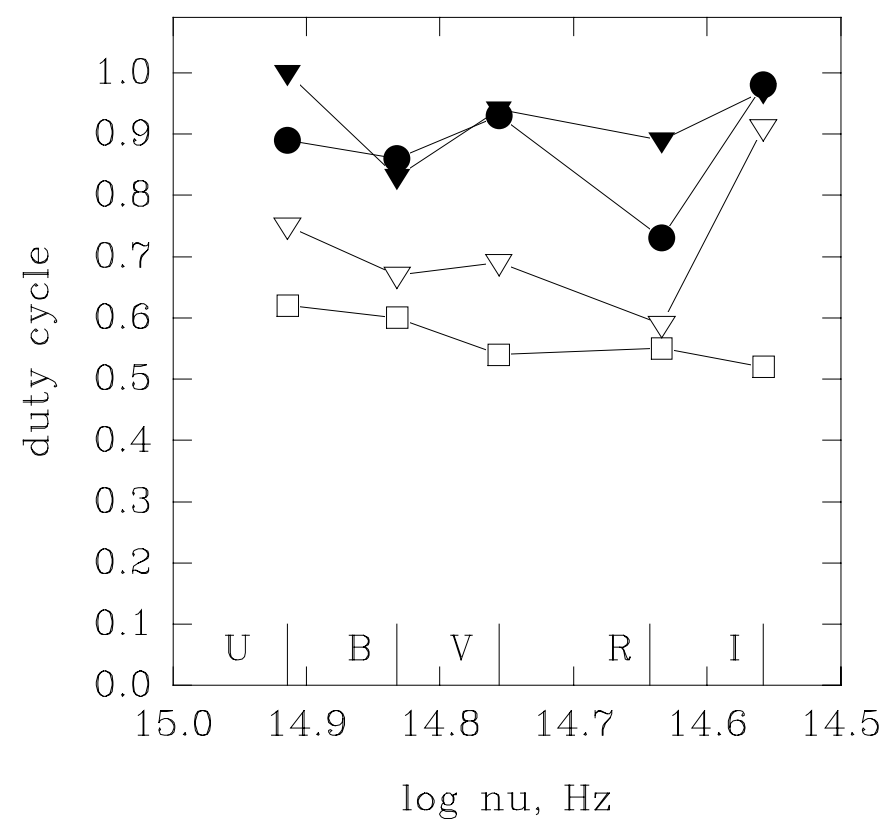

Fig. 16. "Duty cycles" for NGC 5548 (filled circles), NGC 7469 (open triangles), NGC 4151 (open squares), and NGC 1275 (filled triangles). See text. The locations of $U B V R I$ wave bands are given by vertical lines in the abscissa.

\subsection{Discussion}

The long-term variability of the NGC 5548 galaxy nucleus is consistent with the light curve obtained at $5100 \AA$ by Peterson et al. (1999). Some observations discussed in the present paper, were analyzed earlier, together with others obtained during the AGN Watch campaign in 1995 (see Table 9 in the paper by Korista et al. 1995), and during the short-term monitoring campaign in June 1998 (Dietrich et al. 2001). 
It is generally assumed that the prime energy source of an AGN is a massive $\left(\geq 10^{6} M_{\odot}\right)$ black hole with a surrounding accretion disc. Several recent results support this model of the central engine of AGN (Stirpe 1996). The accretion disc is considered to be responsible for part or all of the continuum emission and possibly for part of the line emission. Intranight microvariability is explained easily in the frame of this theory as instabilities on the accretion disc (DI). As the variability of blazars is due to accretion processes, Elliot \& Shapiro (1974) forecast a time scale of the microvariability of the nicleus of NGC 1275 of the order of $5 \mathrm{~min}$. For this galaxy Edelson \& Malkan (1986) calculated a lower limit of the Schwarzschild radius equal to $3-10$ light minutes. Flux variability may occur on such a time scale. It does not contradict our data on intranight variability of the optical continuum flux of all Seyfert galaxies discussed in this paper.

The results may be explained by the standard model where shocks are propagating down a relativistic jet: the shock-in-jet model (SJ). There will be a high degree of microvariability, if jets are bent and turbulent (Marscher \& Travis 1991; Wagner 1991). The explanation of the variability is based on the scenario of shocks propagating within the jet entering a turbulent region.

The main alternative theory by Terlevich et al. (1992) is based on multiple supernova explosions. Rapid variations are not easily explained by this theory.

Therefore, DI and SJ theories are preferable in explaining the intranight microvariability in Seyfert galaxies.

A moderately strong correlation in the sense of greater amplitude of microvariability when OJ 287 (a BL Lac type object) is fainter, has been observed by González-Pérez et al. (1996); one would expect such a correlation if all microvariability were generated by the same mechanism and typically involved the same level of energy generation (e.g. flickering on the accretion disc with a constant energy output), independent of background level. No strong dependence of flux on the amplitudes of intranight variability was found. No correlation was found in the case of NGC 7469 (Merkulova 2000), either. Thus, one can conclude that there is a discrepancy between the model of Disc Instability (DI) and the intranight variability manifestation.

Heidt \& Wagner (1996) studied the intraday variability behavior of a complete sample of radio-selected BL Lac objects. In 28 out of 34 objects ( $82 \%$ ) they detected intraday variability. Assuming that this detection rate could be translated into a duty cycle (the fraction of time, where a BL Lac object is variable), the duty cycle in this sample would be at least 0.8. Using Structure Function analysis, they derived typical timescales for 21 objects that lie in the range between 0.5 and 3 days. The observations can in general be explained with the standard shock-in-jet model, with the exception of the two best sampled BL Lac objects that display a variability behavior that can hardly be explained by this model.
In this paper the comparison of the characteristics of long and short timescale variations of NGC 5548 with those of NGC 4151, NGC 7469 and NGC 1275 is carried out. It was shown, that $U B V R I$ variability may be a result of random processes at different timescales:

a) all galaxies show a mixture of flicker noise and shot noise on timescale of years, excluding NGC 1275, where only flicker noise is observed at all wave bands;

b) the variability on time scales of from days to hundreds of days is characterized by flares with different slopes and different durations;

c) it is supposed that intranight variations, particularly for NGC 5548, are mostly due to shot noise. The intranight variability is transient in character and manifests itself with different probabilities for different galaxies.

Acknowledgements. The author would like to thank Dr. S. G. Sergeev for the software and for stimulating advice; Dr. I. I. Pronik and Dr. S. I. Plachinda for helpful discussions, Dr. L. P. Metik for help during observations, The research described in this publication was made possible in part by Award No. UP1-2116 of the U. S. Civilian Research \& Development Foundation for the Independent States of the Former Soviet Union (CRDF).

\section{References}

Blandford, R. D., \& McKee, C. F. 1982, ApJ, 255, 419

Capriotti, E. R., Foltz, C. B., \& Peterson, B. M. 1982, ApJ, 251,35

Carini, M. T., Miller, H. R., Noble, J. C., et al. 1991, AJ, 101, 1196

Clavel, J., Reichert, G. A., Alloin, D., et al. 1991, ApJ, 366, 64 Deych, A. N. 1966, in Abstracts of the Symposium Instability Phenomena in Galaxies, Byurakan, Armenia

Dumont, A.-M., Collin-Soufrin, S., \& Nazarova, L. S. 1998, A\&A, 331, 11

Dietrich, M., Bender, C. F., Bergmann, D. J., et al. 2001, A\&A, 371,79

Dultzin-Hacyan, D., Schuster, W. J., Parrao, L., et al. 1992, AJ, 103, 1769

Edelson, R. A., \& Malkan, M. A. 1986, ApJ, 308, 59

Elliot, J. L., \& Shapiro, S. L. 1974, ApJ, 192, L3

Fahlmann, G. G., \& Ulrich, T. J. 1975, ApJ, 201, 277

González-Pérez, J. N., Kidger, M. R., \& de Diego, J. A. 1996, A\&A, 311, 57

Heidt, J., \& Wagner, S. J. 1996, A\&A, 305, 42

Hufnagel, B. R., \& Bregman, J. N. 1992, ApJ, 386, 473

Hughes, P. A., Aller, H. D., \& Aller, M. F. 1992, ApJ, 396, 469

Koratkar, A. P., \& Gaskell, C. M. 1991, ApJS, 75, 719

Korista, K. T., Alloin, D., Barr, P., et al. 1995, ApJS, 97, 285

Kunkel, W. E. 1967, AJ, 72, 1341

Lainela, M., \& Valtaoja, E. 1993, ApJ, 416, 485

Lyuty, V. M. 1972, Astron. Zhurn., 49, 930

Lyuty, V. M., Aslanov, A. A., Volkov, I. M., et al. 1989, Pis'ma v Astron Zh. 15, 579 (English transl. Soviet Astron. Lett., $15,247)$

Marscher, A. P., \& Travis, J. P. 1991, in Variability of Active Galactic Nuclei, ed. R.H. Miller, \& P. Wiita (Cambridge Univ. Press), 153

Merkulova, N. I. 2000, AJ, 119, 631 
Merkulova, N. I., Pronik, I. I., \& Metik, L. P. 1999, AJ, 117, 2177

Merkulova, N. I., Metik, L. P., \& Pronik, I. I. 2001, A\&A, 374 , 770

Neckel, Th., \& Chini, K. 1980, A\&AS, 39, 411

O'Dell, S., Puschell, J., Stein, W., et al. 1978, ApJS, 38, 267

Peterson, B. M., Barth, A. J., Berlind, P., et al. 1999, ApJ, 510,659

Piirola, W. 1973, A\&AS, 27, 383

Press, W. H. 1978, Comm. Astrophys., 7, 103

Pronik, I. I., Merkulova, N. I., \& Metik, L. P. 1999a, AJ, 117, 2141

Pronik, I. I., Merkulova, N. I., \& Metik, L. P. 1999b, A\&A, 351, 21

Rodriguez-Pascual, P. M., Alloin, D., Clavel, J., et al. 1997, ApJS, 110, 9

Sergeev, S. G., Pronik, V. I., Malkov, Yu. F., et al. 1997, A\&A, 320,405
Sergeev, S. G., \& Merkulova, N. I. 2002, in preparation

Simonetti, J. H., Cordes, J. M., \& Heeschen, D. S. 1985, ApJ, 296, 46

Stirpe, G. M. 1996, Mem. Soc. Astr. Ital., 67, 1065

Smith, A. G., Nair, A. D., Leacock, R. J., et al. 1993, AJ, 105, 437

Takalo, L. O., Sillanpaa, A., Pursimo, T., et al. 1996, A\&AS, 120,313

Terrel, J., \& Olsen, K. H. 1970, ApJ, 161, 399

Terebizh, V. Yu. 1993, Time Series Analysis in Astrophysics (Nauka Publication, Moskva), 389

Terlevich, R., Tenorio-Tagle, G., Franco, J., et al. 1992, MNRAS, 255, 713

Ulrich, M.-H., Maraschi, L., \& Urry, C. M. 1997, ARA\&A, 35, 445

Wagner, S. J. 1991, in Variability of Active galaxies, ed. W. J. Dusche, S. J. Wagner, \& M. Camenzind, Proc. Heidelberg (Germany, Springer-Verlag), 163 